\title{
Operator's Guide for VAXGAP, a Gamma-Ray Spectrum Analysis Package
}

\author{
E. Wayne KIIllan \\ Douglas A. Fomec
}

Pubilshed August 1992

\section{Idaho National Engineering Laboratory \\ EG\&G Idaho, Inc. Idaho Falls, Idaho 83415}

Prepared for the U.S. Department of Energy Assistant Secretary for Nuclear Energy Under DOE Idaho Fleld Otilce Contract DEnAC07-761001570

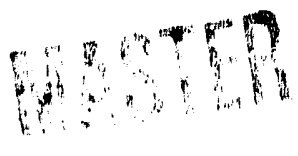




\begin{abstract}
This report describes the gamma-ray analysis program VAXGAP, which has continually evolved at the Idaho National Engineering Laboratory over a period of several years. it is an integrated suite of computer programs for performing analyses of pulse-height spectra from high-resolution gamma-ray spectrometers and assorted support functions. VAXGAP programs operate on Digital Equipment Corporation (DEC) VAX computers running the VMS operating system, and were written in VAX FORTRAN and DEC Digital Command I anguige (DCL). ${ }^{8}$ These prograrns make use of DEC GKS and ReGIS for graphical output on standard terminals and printers, and DEC.windows for graphics on workstations and terminals that support the $X$ Window System protocol. ${ }^{a}$ This report addresses the use of VAXGAP for data acquisition and control, energy scale calibration, and real-time analyses of backgmound and sample pulse-height spectra. Also addressed are the creation and use of efficiency tables and isotope libraries, manipulation of spectrum files and their contents, and graphical display of on-going acquisitions, saved spectra, and mathematical fits to spectral peaks.
\end{abstract}

a. DEC, VAX, VMS, VAX FORTRAN, Digital, ReGIS, and DECwindows are tradem "iks of Digital Equipment Corporation. X Window System is a trademark of the Massachusetts Institute of 'Technology. 


\section{FOREWORD}

This document describes the procedures and commands for using the standard version of VAXGAP. Another document, "Operator's Guide for Using INEL.Specific VAXGAP Programs," EGG-PHY-10338, explains how to use several additional VAXGAP analysis programs that were created specifically for use at the Idaho National Engineering Laboratory (INEL). These INEL-specific programs perform specialized operations and produce specialized output. For a description of VAXGAP analytical models and algorithms, see "VAXGAP: A Code for the Routine Analysis of Gamma-Ray Pulse-Height Spectra on a VAX Computer," EGG-2533. 


\section{CONTENTS}

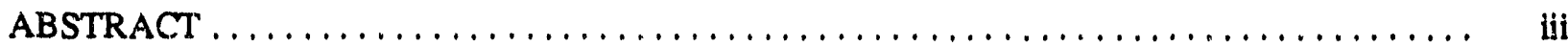

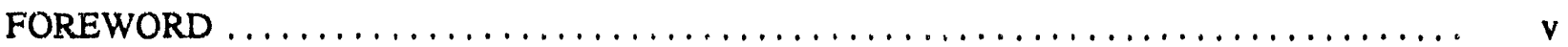

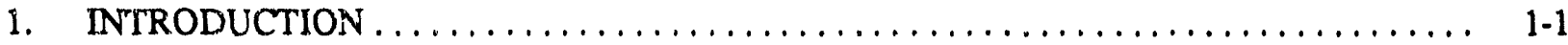

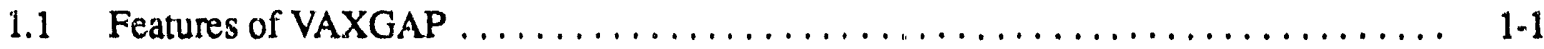

1.2 Computer Peripherals Required to Run VAXGAP $\ldots \ldots \ldots \ldots \ldots \ldots \ldots \ldots \ldots, 1-1$

1.3 Notation Standards $\ldots \ldots \ldots \ldots \ldots \ldots \ldots \ldots \ldots \ldots \ldots \ldots \ldots \ldots \ldots \ldots$

2. ACCUMULATING AND TRANSFERRING MULTI-CHANNEL ANALYZER DATA . . . . 2-1

2.1 Define AIM Ethernet Addresses $\ldots \ldots \ldots \ldots \ldots \ldots \ldots \ldots \ldots \ldots \ldots \ldots \ldots \ldots$

2.2 Start and Stop Data Accumulation $\ldots \ldots \ldots \ldots \ldots \ldots \ldots \ldots \ldots \ldots \ldots \ldots \ldots \ldots \ldots$

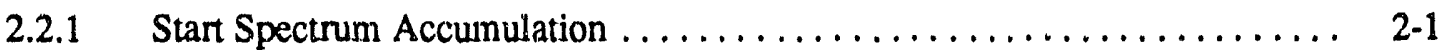

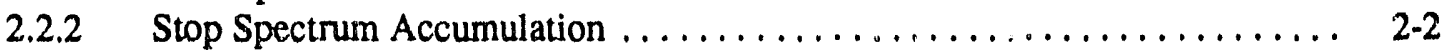

2.2.3 Avoid Errors While Starting and Stopping $\ldots \ldots \ldots \ldots \ldots \ldots \ldots \ldots .2-2$

2.3 Transfer Spectral Data from the Detector System to SLOT\$SPC . . . . . . . . . . 2-2

2.4 Obtain Status Information $\ldots \ldots \ldots \ldots \ldots \ldots \ldots \ldots \ldots \ldots \ldots \ldots \ldots \ldots \ldots$

3. PERFORMING GENERAL SPECTRUM ANALYSIS $\ldots \ldots \ldots \ldots \ldots \ldots \ldots \ldots \ldots \ldots$

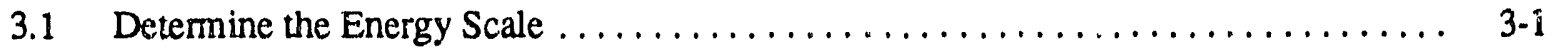

3.1.1 Use Known Peak Positions and Energies (UCLB) $\ldots \ldots \ldots \ldots \ldots \ldots \ldots . . .1$

3.1.2 Use a Thorium Spectrum (THOR) ..................... 34

3.1.3 Use Pulser Peaks from the EG\&G Idaho Precision Pulser (PEQ) ....... $3-5$

3.1.4 Store and Manipulate the Calibration File $\ldots \ldots \ldots \ldots \ldots \ldots \ldots \ldots . . .5$

3.2 Enter Spectrum Parameters (HEAD) $\ldots \ldots \ldots \ldots \ldots \ldots \ldots \ldots \ldots \ldots \ldots \ldots \ldots$

3.3 Analyze Background Spectra (BACK) $\ldots \ldots \ldots \ldots \ldots \ldots \ldots \ldots \ldots \ldots \ldots$

3.4 Perform General Spectrum Analysis $\ldots \ldots \ldots \ldots \ldots \ldots \ldots \ldots \ldots \ldots \ldots \ldots \ldots \ldots \ldots \ldots \ldots$

3.4.1 Analyze Spectra Using GAP ......................... 3-12

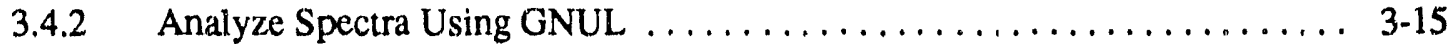

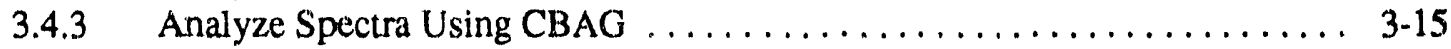

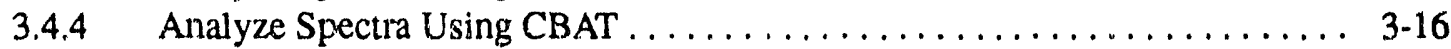

3.5 Construct Parent-Daughter Files $\ldots \ldots \ldots \ldots \ldots \ldots \ldots \ldots \ldots \ldots \ldots \ldots \ldots \ldots \ldots$

4. OPERATING MISCELLANEOUS PROGRAMS $\ldots \ldots \ldots \ldots \ldots \ldots \ldots \ldots \ldots \ldots \ldots$ 
4.1 Create and Modify Efficiency Curves/Tables (EFF) $\ldots \ldots \ldots \ldots \ldots \ldots \ldots \ldots \ldots$

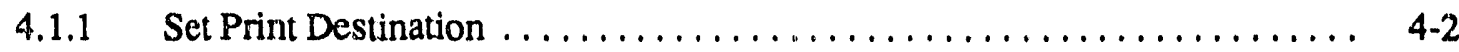

4.1.2 Analyze Reference Standard Spectrum .................. 4-2

4.1.3 Generate Table of Experimental Results .................. 4-2

4.1.4 Fit Curve on X-Window Terminal or VAX Workstation $\ldots \ldots \ldots \ldots \ldots .4 .4$

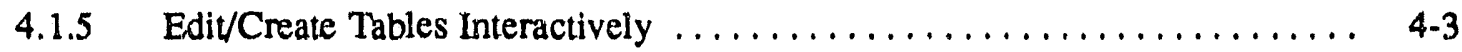

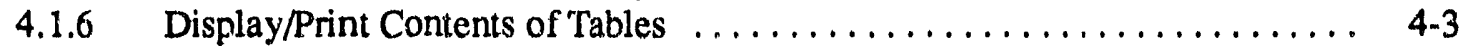

4.1 .7 Plot Efficiency Tables $(\log$ Plot $) \ldots \ldots \ldots \ldots \ldots \ldots \ldots \ldots \ldots \ldots \ldots \ldots \ldots$

4.1 .8 Plot Efficiency Tables (Linear Plot) . . . . . . . . . . . . . . . . . . . 4-3

$\$ 1.9$ Plot Efficiency Tables (EFF*ENG**-A) $\ldots \ldots \ldots \ldots \ldots \ldots \ldots \ldots \ldots, 4-3$

4.1 .10 Print Sorted List of Tables $\ldots \ldots \ldots \ldots \ldots \ldots \ldots \ldots \ldots \ldots \ldots \ldots \ldots, 4.4$

4.1.11 Test Interpolation of Tables .......................... 44

4.1.12 Edit/Create Reference Standard Data Sheet .................. $4-4$

4.2 Create VAXGAP Isotope Libraries (MAKE_LIB) $\ldots \ldots \ldots \ldots \ldots \ldots \ldots \ldots$

4.2.1 Create the Parent-Daughter File $\ldots \ldots \ldots \ldots \ldots \ldots \ldots \ldots \ldots \ldots \ldots, 4.5$

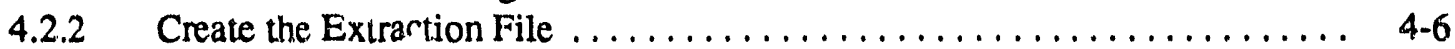

4.2 .3 Create the Add-Delete File $\ldots \ldots \ldots \ldots \ldots \ldots \ldots \ldots \ldots \ldots \ldots \ldots .4 .4$

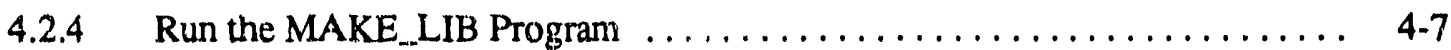

4.2 .5 Run the NIL Program $\ldots \ldots \ldots \ldots \ldots \ldots \ldots \ldots \ldots \ldots \ldots \ldots \ldots . \ldots \ldots$

4.2 .6 Examine the RGH.LOG File $\ldots \ldots \ldots \ldots \ldots \ldots \ldots \ldots \ldots \ldots \ldots \ldots \ldots, 4-10$

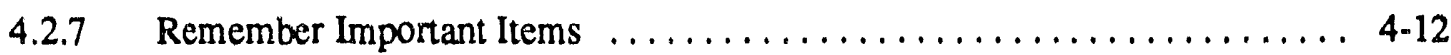

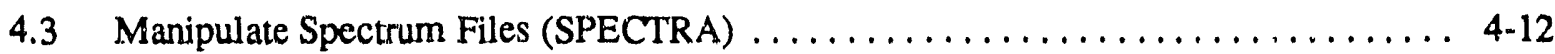

4.3.1 Read from TK70 or TK50 Cartridge Tape ................. 4-13

4.3 .2 Write to TK70 ............................ 4-14

4.3.3 Transfer Spectra from Save Area to SLOT\$SPC ............... 4-16

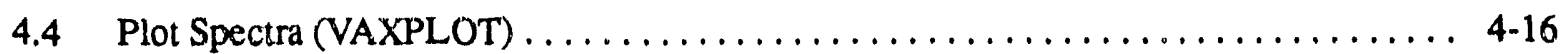

4.5 Edit the Spectrum Parameter Block (EPB) $\ldots \ldots \ldots \ldots \ldots \ldots \ldots \ldots \ldots \ldots$

4.6 Edit the Spectrum Slot Calibration File (ECB) $\ldots \ldots \ldots \ldots \ldots \ldots \ldots \ldots \ldots \ldots \ldots$. $\ldots \ldots$

4.7 Print the Spectrum Header (PRHEAD) $\ldots \ldots \ldots \ldots \ldots \ldots \ldots \ldots \ldots \ldots \ldots \ldots \ldots \ldots \ldots$

5. OPERATING MISCELLANEOUS PROGRAMS ON X-WINDOW WORKSTATIONS $\ldots \ldots \quad 5-1$

5.1 Generate Efficiency Curves (ECF) $\ldots \ldots \ldots \ldots \ldots \ldots \ldots \ldots \ldots \ldots \ldots \ldots \ldots \ldots \ldots$

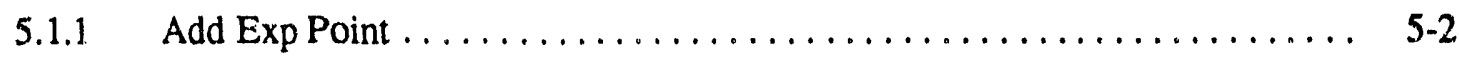

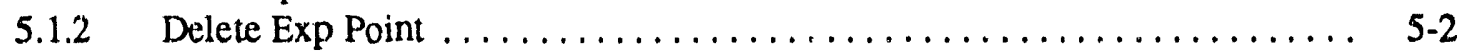

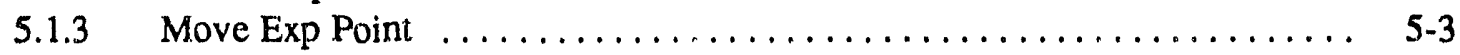

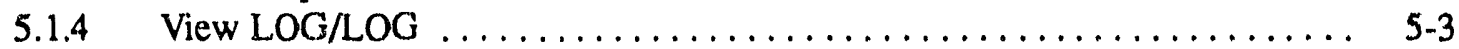

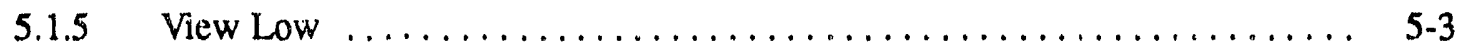

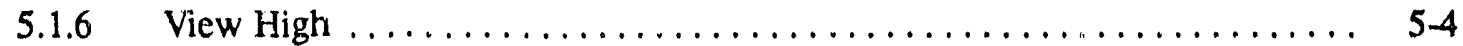

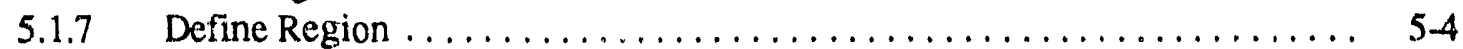

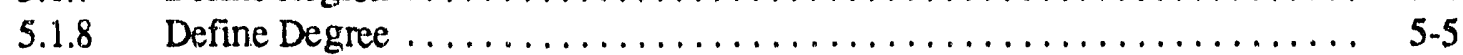




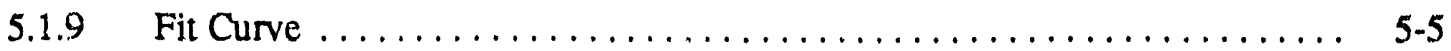

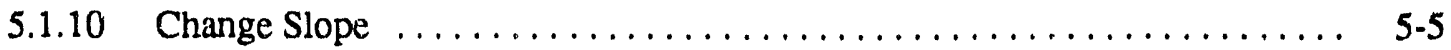

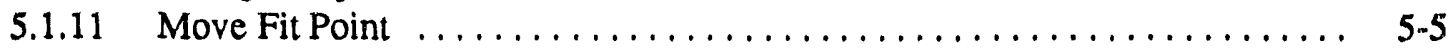

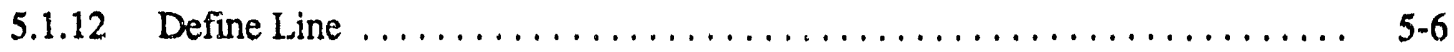

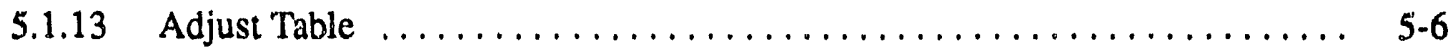

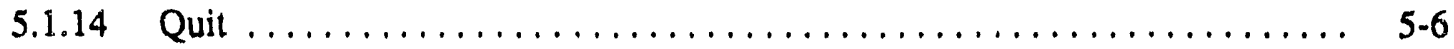

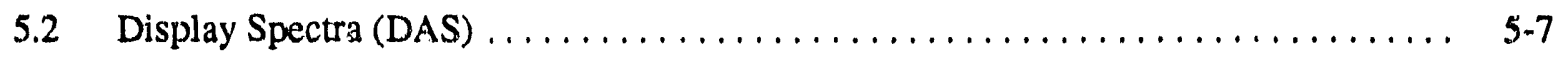

5.2.1 Change the Size of the Display $\ldots \ldots \ldots \ldots \ldots \ldots \ldots \ldots \ldots \ldots \ldots, 5.8$

5.2 .2 Move the Starting Channel $\ldots \ldots \ldots \ldots \ldots \ldots \ldots \ldots \ldots \ldots \ldots \ldots \ldots, 5,8$

5.2 .3 Identify Nuclides $\ldots \ldots \ldots \ldots \ldots \ldots \ldots \ldots \ldots \ldots \ldots \ldots \ldots$

5.3 Fit Peaks Interactively and Display $\operatorname{Spectra}(\mathrm{GINA}) \ldots \ldots \ldots \ldots \ldots \ldots \ldots$

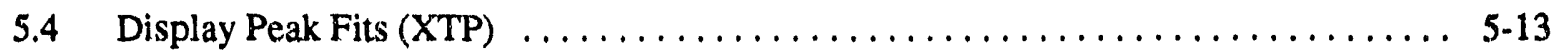

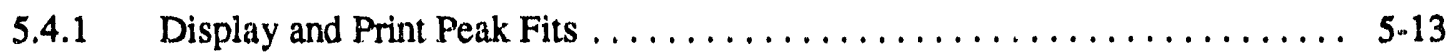

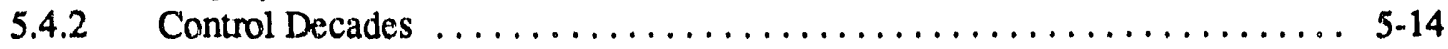

Appendix A-Command Summary for VAXGAP $\ldots \ldots \ldots \ldots \ldots \ldots \ldots \ldots \ldots \ldots \ldots \ldots$

Appendix B-Logical Assignments in VAXGAP $\ldots \ldots \ldots \ldots \ldots \ldots \ldots \ldots \ldots \ldots \ldots \ldots$

Appendix C-Spectrum Parameter Block Contents $\ldots \ldots \ldots \ldots \ldots \ldots \ldots \ldots \ldots \ldots \ldots \ldots$

Appendix D-File Structure for Ge Spectra $\ldots \ldots \ldots \ldots \ldots \ldots \ldots \ldots \ldots \ldots \ldots \ldots \ldots \ldots \ldots \ldots$

Appendix E--Description of "Flag" Values-Printed as Part of Isotope Summary . . . . . . . . E-1

\section{FIGURES}

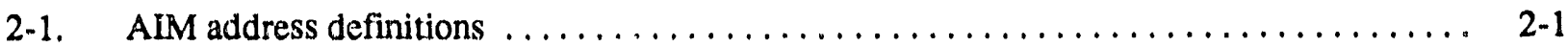

2-2. Example of starting a spectrum accumulation $\ldots \ldots \ldots \ldots \ldots \ldots \ldots \ldots \ldots \ldots \ldots$

2-3. Example of stopping a spectrum accumulation $\ldots \ldots \ldots \ldots \ldots \ldots \ldots \ldots \ldots \ldots \ldots$

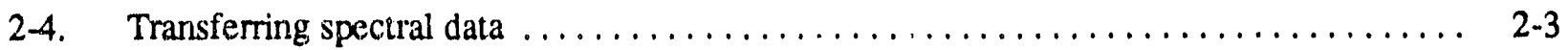

2-5. Status information for each detector system $\ldots \ldots \ldots \ldots \ldots \ldots \ldots \ldots \ldots \ldots \ldots \ldots$

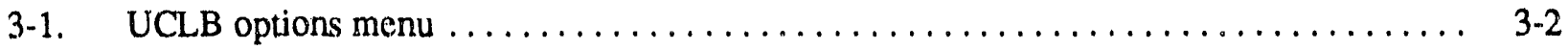

3-2. UCLB spectrum calibration screen $\ldots \ldots \ldots \ldots \ldots \ldots \ldots \ldots \ldots \ldots \ldots \ldots \ldots \ldots \ldots$

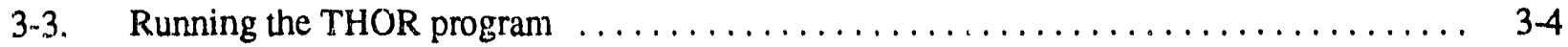

3-4. Running the HEAD program standalone $\ldots \ldots \ldots \ldots \ldots \ldots \ldots \ldots \ldots \ldots \ldots \ldots$ 
3-5. Header input screen $\ldots \ldots \ldots \ldots \ldots \ldots \ldots \ldots \ldots \ldots \ldots \ldots \ldots \ldots \ldots \ldots \ldots \ldots \ldots, \quad 3-6$

3-6. Examples of spectrum file names in save area directories $\ldots \ldots \ldots \ldots \ldots \ldots \ldots \ldots$

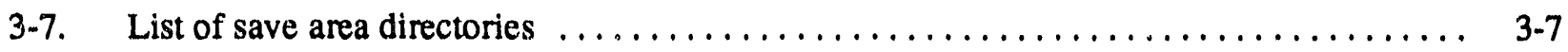

3-8. Example of the BACK input screen $\ldots \ldots \ldots \ldots \ldots \ldots \ldots \ldots \ldots \ldots \ldots \ldots \ldots \ldots$

3-9. Functions performed by the general spectrum analysis routines $\ldots \ldots \ldots \ldots \ldots \ldots$ 3-11

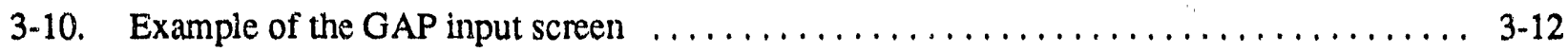

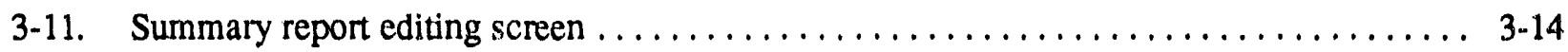

3-12. Background spectra selection menu $\ldots \ldots \ldots \ldots \ldots \ldots \ldots \ldots \ldots \ldots \ldots \ldots \ldots \ldots$

3-1:. Example of the parent-daughter library file $\ldots \ldots \ldots \ldots \ldots \ldots \ldots \ldots \ldots \ldots \ldots$

4-1. Efficiency table maintenance menu $\ldots \ldots \ldots \ldots \ldots \ldots \ldots \ldots \ldots \ldots \ldots \ldots \ldots \ldots \ldots \ldots$

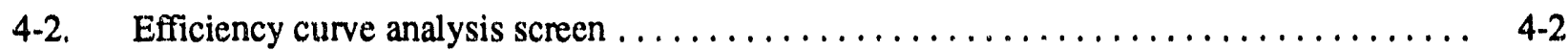

4-3. Example of a parent-daughter ASCII file $\ldots \ldots \ldots \ldots \ldots \ldots \ldots \ldots \ldots \ldots \ldots$

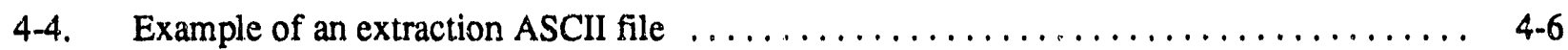

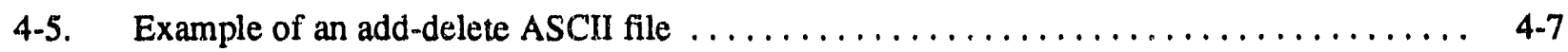

4-6. Running the MAKE_LIB program $\ldots \ldots \ldots \ldots \ldots \ldots \ldots \ldots \ldots \ldots \ldots \ldots \ldots \ldots$

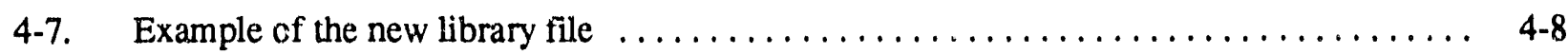

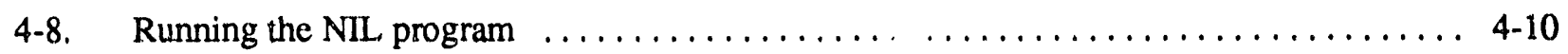

4-9. Example of entries in an RGH.LOG ASCII file $\ldots \ldots \ldots \ldots \ldots \ldots \ldots \ldots \ldots \ldots \ldots$

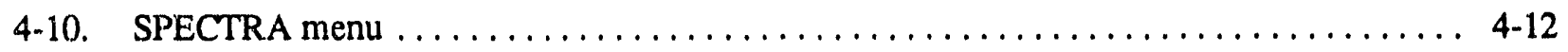

4-11. Interface with SPECTRA for 'Read from TK70' $\ldots \ldots \ldots \ldots \ldots \ldots \ldots \ldots \ldots \ldots$

4-12. Interface with SPECTRA for 'Write to TK'70' $\ldots \ldots \ldots \ldots \ldots \ldots \ldots \ldots \ldots \ldots \ldots . \ldots 4-14$

4-13. Menu to write data to the tape $\ldots \ldots \ldots \ldots \ldots \ldots \ldots \ldots \ldots \ldots \ldots \ldots \ldots \ldots \ldots \ldots$

4-14. Example of transferring spectra from a save area directory to the SLOT\$SPC directory ... 4-16

4-15. Example of the VAXPLOT input screen $\ldots \ldots \ldots \ldots \ldots \ldots \ldots \ldots \ldots \ldots \ldots \ldots \ldots$

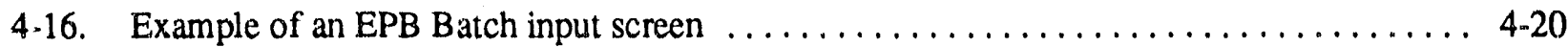

4-17. Example of a calibration block $\ldots \ldots \ldots \ldots \ldots \ldots \ldots \ldots \ldots \ldots \ldots \ldots \ldots \ldots \ldots$ 


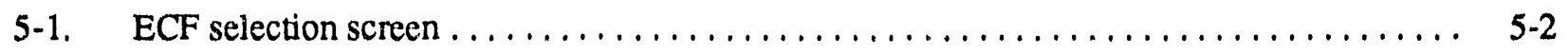

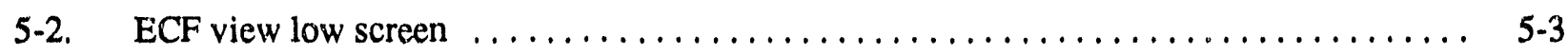

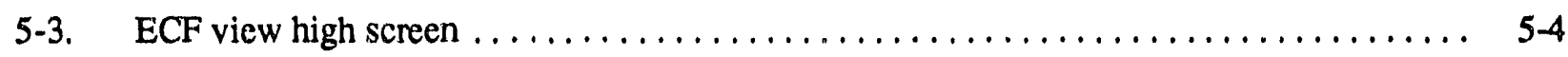

5-4. DAS selection screen $\ldots \ldots \ldots \ldots \ldots \ldots \ldots \ldots \ldots \ldots \ldots \ldots \ldots \ldots \ldots \ldots \ldots$

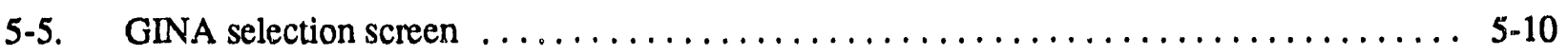

5-6. GINA mark fit screen $\ldots \ldots \ldots \ldots \ldots \ldots \ldots \ldots \ldots \ldots \ldots \ldots \ldots \ldots \ldots \ldots \ldots$

5-7. XTP display screen, showing energy values for all possible peaks $\ldots \ldots \ldots \ldots \ldots \ldots$

5-8. XTP spectrum screen, showing the complete spectrum $\ldots \ldots \ldots \ldots \ldots \ldots \ldots \ldots \ldots$ 


\section{Operator's Guide for VAXGAP, a Gamma-Ray Spectrum Analysis Package \\ 1. INTRODUCTION}

VAXGAP, a gamma-ray pulse-height spectrum analysis software package, is a collection of programs that run on a VAX computer under the VMS operating system in the Radiation Measurements Laboratory (RMI) at the Idaho National Engineering Laboratory. This document describes how to use the VAXGAP software package. In order to use this manual most effectively, you should have a fundamental knowledge of the VMS operating system.

\subsection{Features of VAXGAP}

VAXGAP offers the following features:

- Automatic peak search

- Two different methods for determining ambient background with subsequent radionuclide activity corrections for this background activity

- Computation of radionuclide activities for a set of nuclides that you select

- Multi-line analysis with weighted averaging and outlier elimination to determine concentration values

- Parent-daughter corrections performed to the grandparent level

- Completely automatic energy-scale determination using a Th-228 calibration spectrum

- Routines for the analysis of spectra collected on spectrometer systems equipped with EG\&G Idaho's special electronic pulser injection technique for spectrum-specific determination of gain and zero, real-time spectrometer monitoring, random summing, and dead-time corrections

- Interactive editing of analysis summary sheets to eliminate erroneous entries

- Spectral data manipulation routines for piotting spectra on hardcopy plotters or on operator terminals (CRTs); spectral data storage and retrieval from disk or magnetic tape

- Special interactive photopeak fitting and efficiency curve generation on X-Window terminals

- Control of Canberra Industry's Acquisition Interface Module (AIM) for spectral data colleccion

- Selectable units for quantity and activity to allow you to tailor output for many different types of radioactive samples such as charcoal filters, liquid samples, gas sample cylinders.

\subsection{Computer Peripherals Required to Run VAXGAP}

Although the basic spectral analysis programs will run on any VAX computer with the VMS operating system, a few of the VAXGAP functions described in this manual require additional or particular types of peripherals. The following list shows the functions that require special equipment: 
- Direct spectrometer control is designed for a Cánberra AIM multi-channel analyzer (MCA). Since the AIM MCA is an Ethernet device, the computer must be equipped with end node DECnet.

- Terminal spectral plotting requires a DEC VT330 or equivalent.

- Hardcopy spectral plotting requires a Digital LN03 Plus laser printer or equivalent.

- Graphics programs require an X-Window terminal or VAX workstation.

\subsection{Notation Standards}

The following notation standards are used throughout this manual:

- In examples, comments are prefaced with an exclamation mark ' $!$. These comments are only for clarification of the example. Do not type the '!' or the comments when entering commands.

- The 'Return' key' is denoted by '<cr>'.

- The notation ' $<C t r l \mathrm{Z}>$ ' means to hold down the 'Ctrl' key and press ' $\mathrm{Z}$ '.

- Except in figures, single quotation marks (') show the command or response you should type. Do not type the quotation marks with your entry. (See Appendix A for a list of commands that are available on the standard version of VAXGAP.)

- A name in all uppercase letters with a dollar sign (\$) (e.g., GAP\$EXE) denotes a logical (which points to a directory) where files are stored (see Appendix B for a list of the logical assignments made in the standard VAXGAP environment). 


\section{ACCUMULATING AND TRANSFERRING MULTI-CHANNEL ANALYZER DATA}

This section explains how to start or stop data accumulation, transfer spectral data from the analyzer to a slot in the storage directory', obtain status information f!om all detector systems, and enter acquisition interface module (AIM) addresses.

To control a Canberra AIM, you may enter commands from any terminal at the DCL level. If any command is incomplete, VAXGAP will ask for additional input.

\subsection{Define AlM Ethernet Addresses}

Since each AIM is an Ethernet device, it has a unique, six-digit Ethemet address assigned to it. In order to connect a new AIM to the computer network, you need to define its address in VAXGAP either by entering a DCL command $O$. by saving address definitions in the SYLOGIN.COM file, which is located in the SYS\$MANAGER directory. If you use the SYLOGIN.COM fide, the address definitions will be made known to eny user who logs on to the system.

To define an Ethernet address, use VMS global symbois in the form dd\$AIM, where dd is the detector system designation. Eachi global symbol defines two detector systems. For example, the symbol A1\$AIM defines detector systems $A 1$ and $A 2$. The subsequent symbol for an " $A$ " detector wnuld be $A 3 \$ \mathrm{~A} I M$, which would define detector systems A3 and A4. Figure 2-1 sliows an example of three AIMs with their corresponding addresses and detectors

$$
\begin{array}{ll}
\text { DI\$AIM: }==" 00013 F " & \text { !Detector } 1 \text { and } 2 \\
\text { D3\$AIM: }==" 000070 " & \text { !Detector } 3 \text { and } 4 \\
\text { D5\$AIM: }==\text { "0007E4" } & \text { !Detector } 5 \text { and } 6
\end{array}
$$

Figure 2.1. AlM address definitions.

After you define the symbols, VAXGAP will be ready to accept commands for those detector systems. For example, if you enter a command to siart detector D4, VAXGAP will evaluate the D3\$AIM symbol (because detectors D3 and D4 are controlled by the D3\$AIM module) and perform the action on detector 2 on that AIM.

\subsection{Start and Stop Data Accumulation}

\subsubsection{Start Spectrum Accumulation.}

- Enter the START command in the form 'STA <detector> <live-time><real-time>' and press $\langle c r\rangle$.

$<$ Detector is the two-letter ID of the MCA letter and the detector number (e.g., B3). <Livetime> is the preset live time (or zero if none), and <real-time> is the preset real time (again zero if none).

The command 'STA B'3 3600 0' would start detector system B3 on the AIM whose address is defined by the symbol B 3 SAIM, with a live time preset of one hour. (In subsequent examples, the detector system may 
be referred to as a detector slot.) Figure 2-2 shows an example of two ways to enter the START commandyou can enter the entire command at once or enter it on! line at a time.

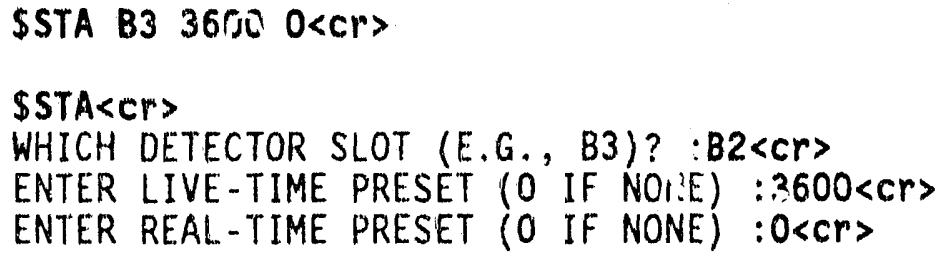

Figure 2-2. Example of starting a spectrum accumulation.

\subsubsection{Stop Spectrum Accumulation.}

- Enter the STOP command in the form 'STO <detecto $>$ ' and press <cr

Again, <detector> represents the two-letter ID of the AIM symbol and the detector number (e.g., B3).

Figure 2-3 shows two ways to enter the STOP command.

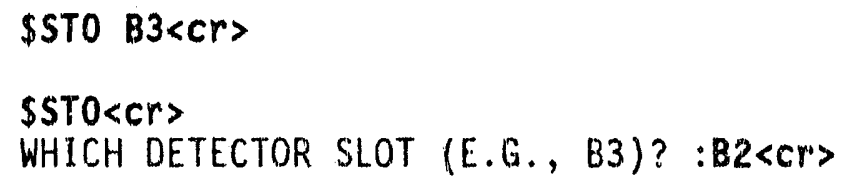

Figure 2-3. Example of stopping a spectrum accumulation.

2.2.3 Avold Errors While Starting and Stopplng. If the previous accumulation has timed out, you do not need to enter the STOP co imand before starting another accumulation. However, if the previous accumulation has not timed out, yc u must enter the STOP command on that slot before starting another accumulation.

\subsection{Transfer Spectral Data from the Detector System to SLOT\$SPC}

After you stop data accumulation, you need to transfer the data to the directory pointed to by the logical SLOT\$SPC, where the data will be temporarily stored in files (specirum slots) having the format dd.DAT. The first $d$ denotes the MCA, and the second $d$ indicates the detector connected to the MCA. For example, A1.DAT contains a spectrum collected on detector system A1, and C4.DAT contains a spectrum collected on decector system $\mathrm{C} 4$.

- Enter the $\$ 90$ command in the form ' $\$ 90<$ detector>' and press <cr>.

If the live time is zero, VAXGAP will not transfer data and will display an error message.

Figure 2-4 shows an example of the $\$ 90$ command. 
$\$ S 90 \quad B 3<C r>$

TOTAL COUNTS IN SPECTRUM: 75825 . COUNT/LIVE TIME (SECONDS): 252.8

$\$ \$ 90<\mathrm{cr}\rangle$

WHICH DETECTOR SLOT (E.G., B3)? :B2〈Cr>

TOTAL COUNTS IN SPECTRUM: 100320 . COUNT/LIVE TIME (SECONDS): 315.2

Figure 2-4. Transferring spectral data.

As each spectrum is read from a detector system into a spectrum slot, it inherits the energy scale and width eq'ation parameters that are identified for that spectrum slot. The energy calibration programs UCLB and THOR are used to calculate these calibration parameters (see Sections 3.1.1 arid 3.1.2, respectively). No other programs will calculate values for these parameters. The Edit Calibration Block (ECB) program, however, can be used to manually change the calibration parameters for each spectrum slot (see Section 4.6).

The spectral data slots in SLOT\$SPC are intermediate in nature, i.e., the data contained in a particular slot will be overwritter when a spectrum is transferred from the same MCA and detector. Use the HEAD program to transfer data from the temporary slot files to permanent storage files (see Section 3.2).

\subsection{Obtain Status Information}

- Type 'ADCS' and press <cr>.

The essential status information for each detector system will be displayed. Figure 2-5 is an example of status information.

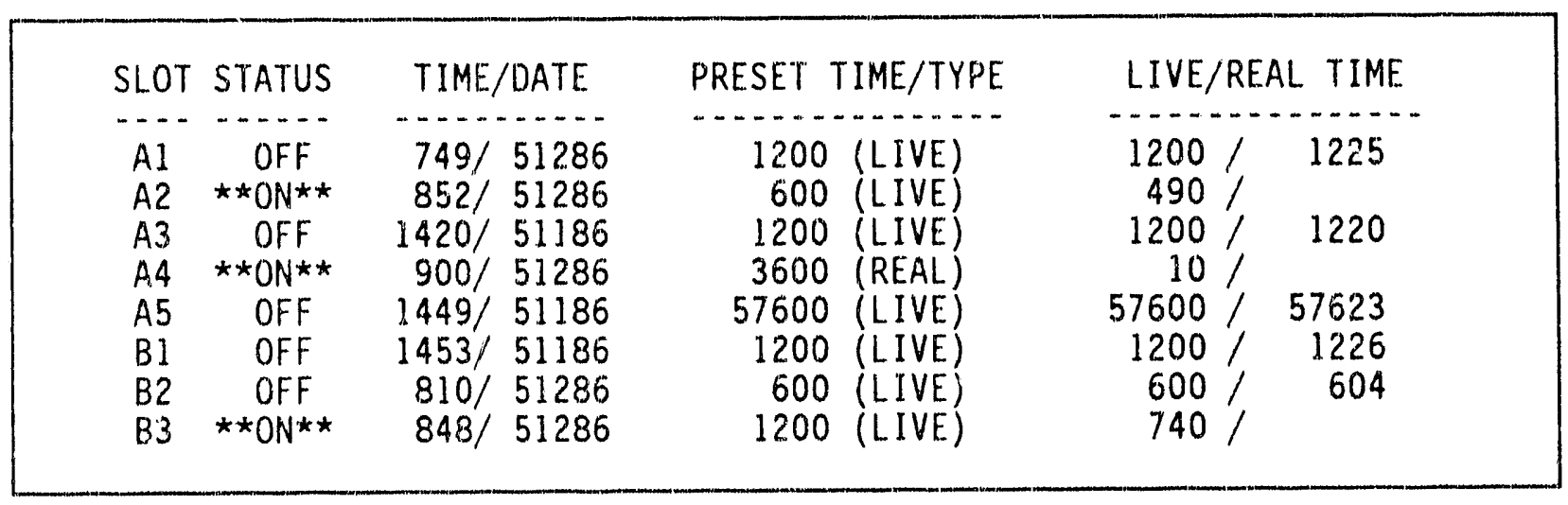

Figure 2-5. Status information for each detector system. 


\section{PERFORMING GENERAL SPECTRUM ANALYSIS}

After you have accumulated spectral data and transferred it to a storage directory (see Section 2), you are ready to perform spectrum analysis. This chapter leads you through the steps necessary to run spectrum analysis routines like GAP, GNUL, CBAG, and CBAT.

In order for a spectrum analysis to be useful, you need to determine the energy scale associated with a detector system. With VAXGAP, you may use three different programs to determine the energy scale (see Section 3.1).

Each spectrum analysis routine invokes the HEAD program, which lets you enter the sampling times, the spectrum ID number, and other spectrum-specific parameters. The HEAD program is the same in all analysis routines and is explained in Section 3.2.

When a spectrum is accumulated, there is alwaj's ambient background radiation that will be measured with the sample's radioactivity. You must subtract this background radiation from the sample spectrum in order to obtain results that reflect the contents of the sample only, particularly when the sample's activity is near background levels. In the VAXGAP environment, you can subtract the ambient background radiation by using two different techniques, which are described in Sections 3.3 and 3.4.

Section 3.4 also explains how to use the spectrum analysis routines GAP, GNUL, CBAG, and CBAT, and Section 3.5 describes how to construct parent-daughter files.

\subsection{Determine the Énergy Scale}

When you transfer a spectrum from an MCA, energy-scale and photopeak-width calibration coefficients are read from a calibration file for that detector system. The calibration files (e.g., A1.CLB, B3.CLB) are located in the directory pointed to by the logical SLOT\$SPC. The information in the calibration file can be modified with three different programs, which determine the energy scale:

- UCLB-determines the energy scale based on operator-supplied peak positions and energies

- $\quad$ THOR-determines the energy scale based on a Th-228 calibration spectrum

- PEQ-determines the pulser energy equivalents from an EG\&G Idaho precision pulser using a Th-228 calibration spectrum.

Sections 3.1.1, 3.1.2, and 3.1.3 explain each of these three programs. Section 3.1.4 describes the calibration file.

3.1.1 Use Known Peak Positlons and Energles (UCLB). The UCLB program determines the energy scale assigned to a spectrum slot by determining the relationship between photopeak positions and corresponding energies. These photopeak positions can point either to an actuai peak in a spectrum located in a slot or to positions of peaks located in a spectrum collected elsewhere and for which the same energy scale is derived. In the first case, in which a spectrum is present, the calibration is called a "spectrum calibration." The other case is called a "non-spectrum calibration."

The ordered pairs of peak position and energy are used to perform a least-squares fit to either a linear energy equation of the form Energy $=\mathrm{A}+\mathrm{BX}$, where $\mathrm{X}=$ the channel position, or to a quadratic equation of the form Energy $=A+B X+C X^{2}$. 
Either type of fit can use weighted or unweighted energy values. If you know the uncertainty in the energy values, you can request a weighted fit, in which each energy value will be weighted according to its associated uncertainty. The weighting function depends on thi type of calibration being performed (spectrum or non-spectrum). Both methods use a weighting factor of $1.0 /$ (uncertainty in energy) ${ }^{2}$.

To run the UCLB program:

- Type 'UCLB' and press <cr>.

The UCLB program will display the UCLB options menu, as shown in Figure 3-1.

\begin{tabular}{|c|c|c|}
\hline \multicolumn{3}{|c|}{$\begin{array}{l}\text { ISUCLB<cr } \\
\begin{array}{l}\text { Enter the spectrum slot }(\mathrm{e} . \mathrm{g} ., \mathrm{N} 1): \text { N } 9<\mathrm{cr}> \\
\text { !The slot in which the calibration } \\
\text { !results will be placed }\end{array}\end{array}$} \\
\hline $\begin{array}{l}\text { THE OPTIONS ARE: } \\
\text { S-Spectrum cal ibration } \\
\text { N-NO spectrum }\end{array}$ & $\begin{array}{l}\text { L-Linear fit } \\
\text { Q-Quadratic fit }\end{array}$ & $\begin{array}{l}\text { W-Weighted } \\
\text { U-Unweighted }\end{array}$ \\
\hline
\end{tabular}

Figure 3-1. UCLB options menu.

- Enter the spectrum slot and press $<c r>$.

The spectrum slot indicates the calibration file where the resulting calibration parameters will be stored.

- Select the calibration options you want by entering one letter from each column and press <cr>.

For example, enter ' $S$ ' for a spectrum calibration, ' $L$ ' for a linear fit, and ' $W$ ' for an energy weighted fit. In Figure 3-1, a non-spectrum calibration with a quadratic, unweighted energy fit was selected.

Sections 3.1.1.1 and 3.1.1.2 describe the different types of input that are required for a spectrum calibration and a non-spectrum calibration, respectively.

3.1.1.1 Callbration Using a Spectrum. Spectrum calibration actually fits the peaks at specified locations in the spectrum to a gaussian function to determine the peak centroids and widths.

If you selected the spectrum calibration option, UCLB will request further information. Figure 3-2 is an example of the type of data UCLB will request.

- Enter the number of peaks to be fit and press $<c r>$.

If you selected a quadratic type fit (see Figure 3-1), then you must enter at least three peak positions and corresponding energies. For a linear fit, you must enter at least two peak positions and energies. 


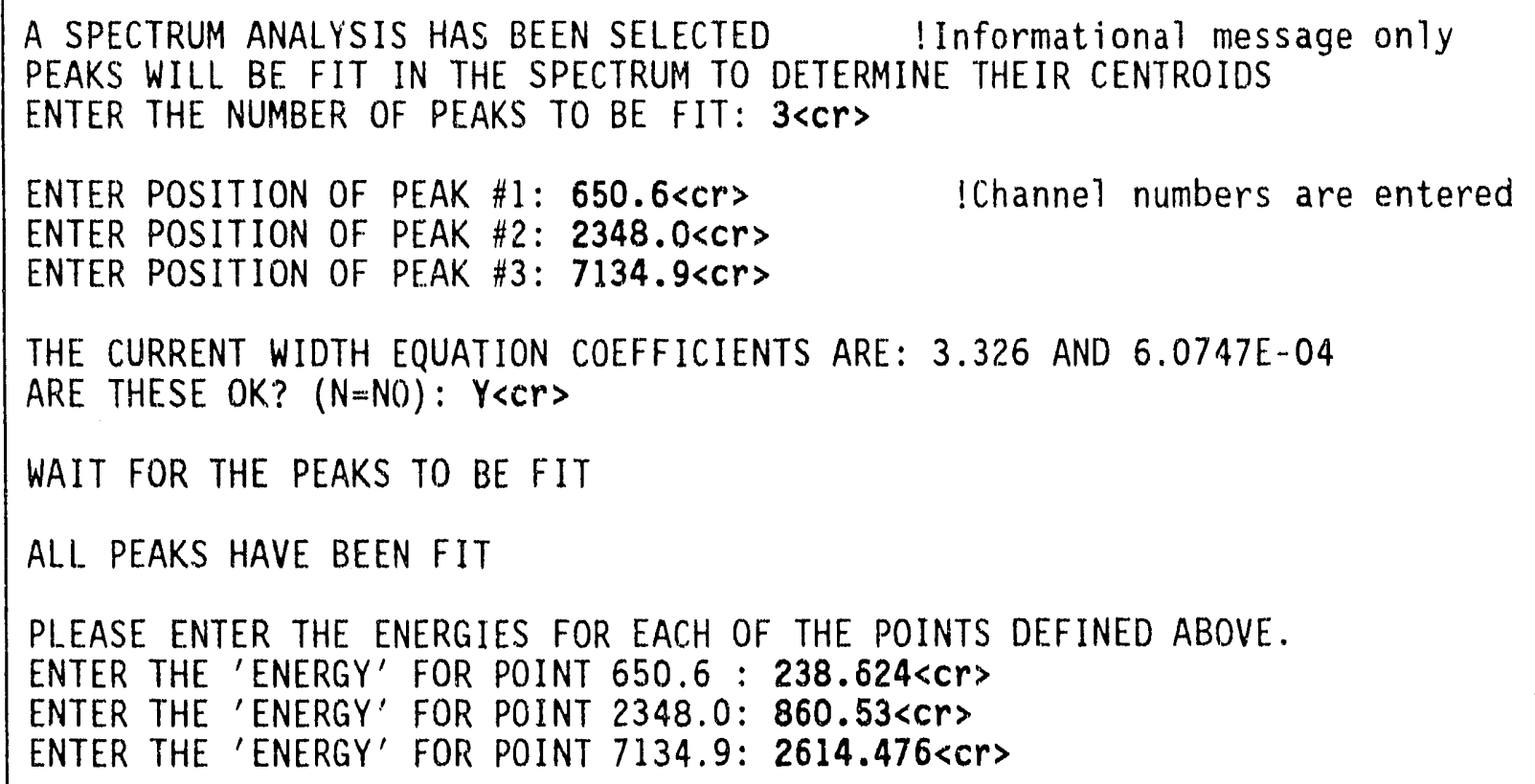

Figure 3-2. UCLB spectrum calibration screen.

- Enter the peak locations in channels (within one channel), pressing $<c r>$ after each entry.

Make sure that the peak positions you enter correspond to the same spectrum slot you entered (see Figure 3-1).

- If the current width equation coefficients are valid, enter ' $Y$ ' and press $<c r>$.

If the coefficients of the width equation are not valid for the current spectrum, you can change them by entering ' $N$ ' and pressing <cr>. UCLB will then let you enter the correct values.

After UCLB has valid width equation coefficients, it will make fits to the peaks. UCLB will then check the peaks to ensure they were fit properly. For each peak that was not fit properly, UCLB will ask you if you want to discard that peak from the set of data points.

- Delete all peaks that were not fit properly.

If you do not delete a badly fit peak, UCLB will not be able to use that data and will quit. Also, if you do delete peaks, but not enough points remain to sufficiently perform the type of fit required (linear or quadratic), UCLB will quit.

- Enter the energies for each of the corresponding peak positions, pressing <cr $>$ after each entry.

If you selected the energy weighted fit (see Figure 3-1), you must also enter the uncertainty associated with the energy.

UCLB will then perform a least-squares fit to the data points and produce coefficient values for the energy and width equations. The spectrum slot is now calibrated. All subsequent spectra read into this slot will have this energy scale. UCLB will display the values for all these parameters and input values on your terminal and will print the values on the line printer. 
When an analysis process ends and output should be printed on the line printer, VAXGAP should display a message like the following on the screen: "Job S000004DA (queue SYS\$PRINT, entry 933) started on SYS\$PRINT." If you do not receive the print message, then the process was not completed or the process was aborted abnormally. To terminate the printing of any job, type 'DEL/ENTRY $=\mathrm{N}$ ', where $\mathrm{N}$ is the entry number (933 in the example shown above).

3.1.1.2 Callbration Without a Spectrum Present. If you selected the non-spectrum calibration option (see Figure 3-1), UCLB will request further information.

- Enter channel positions and their associated widths.

This step is necessary because you will not perform peak fits. UCLB will then proceed as if you were performing a spectrum calibration. The output produced at the end of a non-spectrum calibration is the same as for a spectrum calibration.

3.1.2 Use a Thorlum Spectrum (THOR). You can use the THOR program to automatically determine the coefficients $(A, B, C)$ for the quadratic energy equation, Energy $=A+B X+C X^{2}$, and the coefficients $(Z, Y)$ for the width equation, Width $=Z+Y^{\prime} X$. THOR uses a Th-228 spectrum as a calibration spectrum; from it, THOR automatically calculates the values of the calibration coefficients. THOR then prints a table that shows the values for the coefficients and how well the determined equations fit the data. In general, the process finds the locations of five lines in the spectrum, fits each line to a gaussian function, and performs a least-squares fit of the resulting channel positions to their known energies to obtain the energy equation coefficients. The same least-squares process is repeated using channel positions and full-width-at-hrif-maximum values to determine the coefficients of the width equation. The location of the five knou'n lines depends on two factors:

1. The gain for the spectrometer must be such that the $2614 \mathrm{keV}$ line from the Th-228 spectrum is in the region of 2248-4076 for a $4 \mathrm{~K}$ spectrum and in the region of 4296-8172 for an $8 \mathrm{~K}$ spectrum. Also, the $2614 \mathrm{keV}$ line must have the maximum number of counts over any other line in that region.

2. The zero of the system must be set such that the location of the $238 \mathrm{keV}$ line is less than 50 channels away from the computed channel, $C C$, where $C C=238 /(2614 / X), X$ being the location of the 2614 line.

To use the THOR program:

- $\quad$ Collect a Th-228 spectrum (see Section 2.2).

- Transmit the spectrum to the VAX (see Section 2.3).

- Enter 'THOR' and press <cr.

- Enter the ID of the Thorium spectrum (e.g., A3).

Figure 3-3 shows an example of how to run the THOR program.

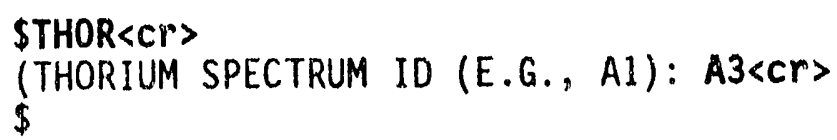

Flgure 3-3. Running the THOR program. 
3.1.2.1 Channel Fitting Errors. If THOR displays the following error message: 'PEAK AT CHANNEL XXX WAS NOT FIT', where XXX is a channel number, it means that THOR found a peak that did not fit and did not modify the coefficients for any of the calibration equations.

3.1.2.2 Incorrect Coefflclent Errors. It is possible, however, for the THOR program to be completed without any error messages, but with coefficients that are completely wrong. This usually happens if the gain/zero of the detector system is off by a large amount. Remember, the gaussian-fitting process that is used to fit the peaks uses the width equation coefficients to determine fitting limits, background values, and other parameters. Therefore, if these values are changed to bad values, the fitting process for subsequent energy calibrations (or spectrum analyses) will never operate correctly.

If this happens, you must change the resulting width equation coefficients before you perform a recalculation. To do this, use the ECB program (see Section 4.6). Also, since the existing coefficients are used as a starting value for the next iteration, you should run THOR at least two times in a row to reset the coefficient values for the width equation back to values that will allow the fitting process to be completed normally.

3.1.3 Use Pulser Peaks from the EG\&G Idaho Precision Pulser (PEQ). For those detector systems equipped with EG\&G Idaho's precision pulser, the energy scale is derived from the position of the pulser peaks and their equivalent energies.

To determine the equivalent energies:

- Use a Thorium spectrum (collected with the pulser peaks).

- Enter 'PEQ' and press <cr>.

- Enter the Thorium spectrum ID and press <cr>.

PEQ uses the same algorithm (and has the same restrictions) as THOR to establish the energy scale. From this energy scale and the position of the pulser peaks in the spectrum, PEQ updates the calibration file with energy scale coefficients and the pulser equivalent energies. "The printed output from PEQ is also the same as THOR, except for an extra two lines that indicate the equivalent energy of the pulsers.

3.1.4 Store and Manlpulate the Callbration Flle. The calibration file (e.g., A1.CLB, B3.CLB) for each detector is stored in the SLOT\$SPC directory. The values in these calibration blocks have been put there by UCLB, THOR, or PEQ. Changes made to the calibration file will have no effect on spectrum files already stored in the spectrum slot file or save area directories. Any spectrum that is subsequently transferred from a detector system will get its calibration coefficients from the corresponding calibration file, and they will be put into the parameter block of the spectrum (see Appendix C). Once a spectrum has been transferred from the detector system, it no longer has any connection to the calibration file. The energy and width calibration will stay with a spectrum file at all times (even on magnetic tape). Thus, you will not need to worry about which calibration is in the detector slot when reanalyzing spectra.

The only way to change the calibration of an existing spectrum file is to run EPB (edit parameter block) and change each coefficient individually (see Section 4.5).

\subsection{Enter Spectrum Parameters (HEAD)}

The HEAD program allows you to enter parameters ('header' information) for each spectrum. Header information describes the sample from which the spectrum was obtained and other counting parameters such as counting geometry and sample times. After you enter information for a spectrum, the HEAD 
program will copy the spectrum file from the spectrum slot file to the appropriate save area directory. If you run the HEAD program once, and then run it again on the same spectrum file, the previous values will be used as defaults.

You may enter spectrum header information in two ivays:

1. Run a spectrum analysis routine such as GAP, GNUL, CBAG, or CBAT (see Section 3.4). Any of these routines will let you invoke the HEAD program and enter header information about a spectrum.

2. Run the HEAD program 'standalone' and enter header information for each spectrum. Then, when you run a spectrum analysis routine on a paricular spectrum, the routine will use that spectrum's existing header information for the analysis.

To run the HEAD program standalone:

- Enter 'HEAD' and press <cr (see Figure 3-4 for an example of the commands).

- Ent'r the name of the spectrum slot (e.g., B4) and press <cr>.

HEAD will display the header input screen, as shown in Figure 3-5.

\section{\$HEAD<Cr> IINITIATE PROGRAM \\ ENTER SPECTRUM FILE NAME: B4<Cr> !ENTER SPECTRUM SLOT}

Figure 3-4. Running the HEAD program standalone.

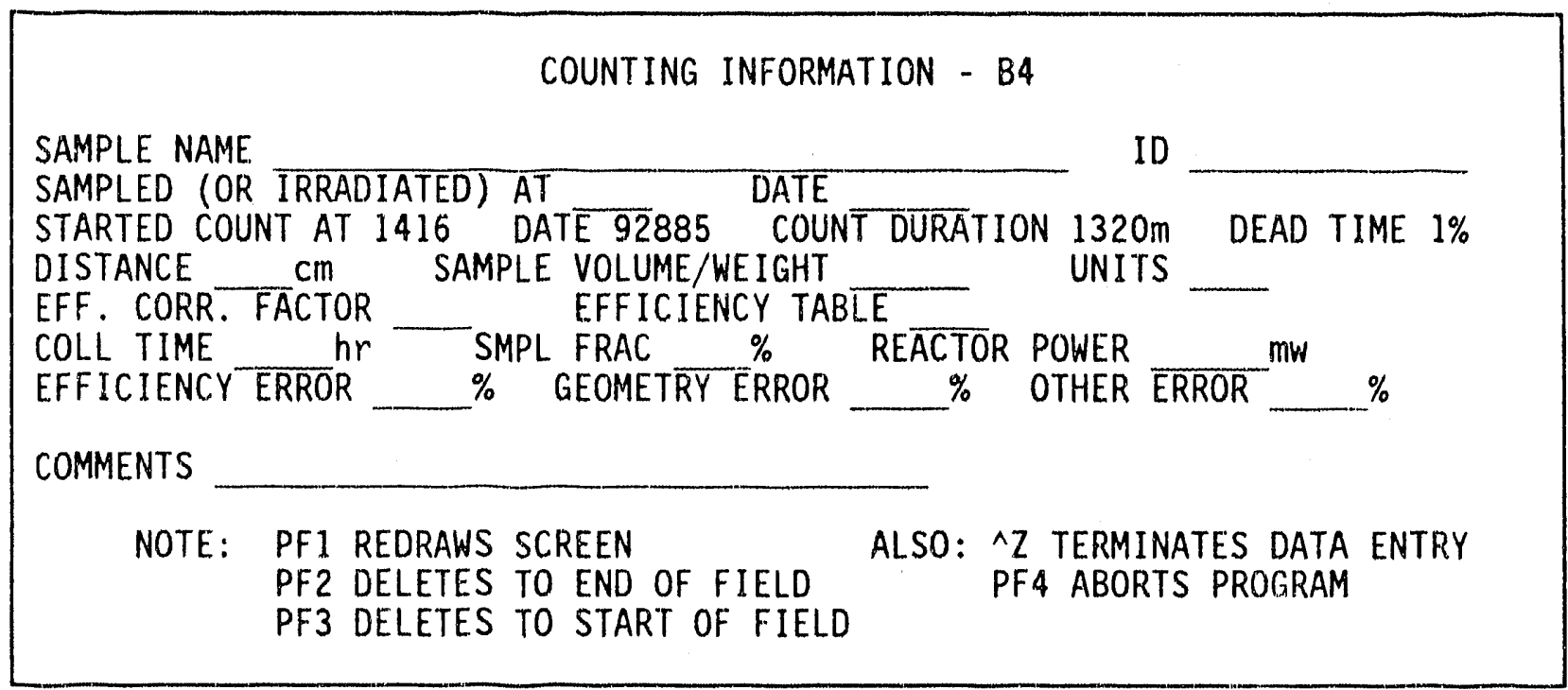

Flgure 3-5. Header input screen. 
- Make entries in the following required fields, pressing <cr> after each entry.

The HEAD program checks many values for validity. If any value is invalid, the HEAD program will display an error message and request that you enter a correct value.

- SAMPLE NAME-a description of the spectrum.

This may be any combination of alphanumeric characters that will fit on the line. The HEAD program will place the sample name in the spectral header block after the ID.

- ID-a unique nine-digit ID followed by a space and a five-digit save area number (for example, 06239203221040 ).

The ID must have the form MMDDYYSSS, where MMDDYY is the month, day, and year, and SSS = sample sequence number for that day. The HEAD program will use this information to name the spectrum file B4MMDDYYSSS.DGE (the first two characters indicate the spectrum slot). Figure 3-6 shows some examples of the various file names you can find in save area directories.

The five-digit save area number must be one of the save area directories shown in Figure 3-7. The HEAD program will copy the spectrum file from the SLOT\$SPC directory to the save area directory that you designate. The SLOT\$SPC directory and the save area directories have the same internal file format, which is described in Appendix D.

File Name

A5021585001.DGE

B1123085021.DGE

\section{Description}

Spectrum taken on MCA A detector \#5 on 15 February 1985, sequence number 001 .

Specimum taken on MCA B detector \#1 on 30 December 1985, sequence number 021 .

Figure 3-6. Examples of spectrum file names in save area directories.

\begin{tabular}{|c|c|c|}
\hline Save Area Number & Save Area & Description \\
\hline 00000 & $\begin{array}{l}\text { GN\$SAVE or } \\
\text { GESSAVE }\end{array}$ & General \\
\hline $\begin{array}{l}01001 \\
01002 \\
01003 \\
01004 \\
02001 \\
03000 \\
04000 \\
05000\end{array}$ & $\begin{array}{l}\text { BK\$SAVE } \\
\text { BW\$SAVE } \\
\text { BS\$SAVE } \\
\text { BF\$SAVE } \\
\text { TH\$SAVE } \\
\text { EF\$SAVE } \\
\text { EN\$SAVE } \\
\text { LP\$SAVE }\end{array}$ & $\begin{array}{l}\text { Instrument Background } \\
\text { Water Background } \\
\text { Soil Background } \\
\text { Filter Background } \\
\text { Thorium Calibrations } \\
\text { Effluents } \\
\text { Environment } \\
\text { Loops }\end{array}$ \\
\hline
\end{tabular}

Flgure 3-7. List of save area directories. 
- SAMPLED (OR IRRADIATED) AT-a lour-digit 24-hour clock time in the form HHMM (for example, 1432).

- DATE-a six-digit date of the form MMDDYY.

- DISTANCE-any integer value (in $\mathrm{cm}$ ).

- SAMPLE VOLUME/WEIGHT-any integer value (see Units below).

- UNITS-for the sample volume/weight (for example, SMPL, CC, ML, L, GAL, GM, or MG).

- EFF. CORR. FACTOR - a real value (integer or floating point).

The HEAD program multiplies this efficiency correction factor with each efficiency value before they are used in spectral analysis aigorithms.

- EFFICIENCY TABLE-any integer value that corresponds to an existing efficiency table.

- Enter information, if desired, in the following optional fields:

- COLL TIME-the sample collection time; any real value (integer or floating point) (in hours).

The HEAD program uses this value to decay correct for the period in which the sample was being gathered, the so-called " $\mathrm{C} 2$ " decay correction.

- SMPL FRAC - the percentage of the sample you are analyzing (use this field only if you are counting a piece of a sample).

- REACTOR POWER-for record keeping and documentation purposes only.

- EFFICIENCY ERROR, GEOMETRY ERROR, and OTHER ERROR-values that will be printed on the report as additional uncertainty in the results.

- COMMENTS-for record keeping and documentation purposes only.

- Press $<C t r l ~ Z>$ to save the information and exit from the HEAD program.

VAXGAP stores the information in the header and parameter blocks of the spectrum and copies the spectrum file from the spectrum slot in the SLOT\$SPC directory to one of the save area directories (TH\$SAVE, BK\$SAVE, GN\$SAVE, etc.).

\subsection{Analyze Background Spectra (BACK)}

In order to obtain a true measure of radioactivity emitted from a sample, you need to subtract the ambient background radiation. In VAXGAP, you may determine this ambient background contribution in two ways:

1. Use the preanalysis technique. Preanalyze the background spectrum and subtract the background photopeak count rate from the corresponding sample photopeak count rate. This is the traditional method. This section describes how to use the BACK program to calculate this value. 
2. Use concurrent background analysis. With this improved method, the regions in the sample spectrum containing peaks of interest are mapped to one or more background spectra to determine the contribution of any existing ambient background activities to the measured sample activities. You may use particular analysis routines to automatically perform these calculations, but these routines are different than the routines that use the output from the BACK program. See Section 3.4 for instructions on running analysis routines and using concurrent background analysis."

The BACK program computes the energies, counts per second, and the uncertainty in the counts-persecond value for all peaks found in the background spectrum. These values are saved in a file (one file for each of the detector slots) in the directory pointed to by the logical SLOT\$BKG. These background results files (e.g., A1.BKG, A2.BKG) are not spectrum files, but contain only the results from a background analysis.

To print any background results file to examine the values currently being used for background subtraction:

- Type 'PRBACK' and press <cr>.

- Enter the ID of the detector system (e.g., B2) for which you want to see the background analysis results.

The PRBACK procedure will print the values.

Each time you run the BACK program, VAXGAP overwrites what was in the background results file for that slot. Consequently, if you run the BACK program on data that do not represent a background spectrum, you must either (a) count a new background spectrum and run BACK on that spectrum, or (b) move the last background spectrum from the spectrum save area (BK\$SAVE) into its appropriate spectrum slot and reanalyze the data. Procedure (b) would work only if the background spectrum calibration coefficients have not changed from when the spectrum was taken.

To run the BACK program:

- Type 'BACK' and press <cr>.

The BACK program will display the BACK input screen (see Figure 3-8 for an example).

If you want to change any of the default values:

- Press $<c r>$ to move the cursor to the value you want to change.

- Enter the new value, which will appear to the right of the current value. For each menu item, only a few values are valid:

- $\quad$ SPECTRUM ID-the spectrum slot (e.g., A1).

a. E. Wayne Killian, Thomas C. Sorensen, "Concurrent Determination of Ambient Background Contribution to Low-Activity High-Resolution Gamma-Ray Spectra," Radioactivity and Radiochemistry, Vol. 3, No. 1, May 1992. 
VAX 'BACKGROUND' ANALYSIS

SPECTRUM ID (e.g., Al): A1

ISOTOPE LIBRARY: MASTER

REQUIRED ENERGY LIBRARY: ZEROLIM

ENTER SPECTRUM HEADER PARAMETERS? YES

PRINT DETAILED PEAK FIT SETUP? NO

PEAK SEARCH SENSITIVITY: 8

Flgure 3-8. Example of the BACK input screen.

- ISOTOPE LIBRARY and REQUIRED ENERGY LIBRARY -may be the name of any compatible library that exists.

Use the directory command with the logicals GE\$ISOTOPE\$LIB and GE\$LIMIT\$LIB to determine which librarits are available.

- ENTER SPECTRUM HEADER PARAMETERS? - either 'YES' or 'NO'.

If you enter 'YES', the HEAD program will nun automatically after you exit the BACK screen (see Section 3.2).

- $\quad$ PRINT DETAILED PEAK FIT SETUP?-also either 'YES' or 'NO'.

If you enter 'YES', VAXGAP will print a detailed output of how each peak was fit, in addition to the normal output.

- PEAK SEARCH SENSITIVITY-a positive integer less than 20.

A smaller value increases the sensitivity of the search. A default sensitivity of 8 is used in all analysis procedures when you do not have the option to enter a different value.

- $\quad$ Press $<c r>$ to replace the old value with the new one.

- Press $<c r>$ again to move the cursor to the next value you want to change. (If the cursor is at the bottom of the screen, press $<\mathrm{cr}>$ to move it to the top.)

When all the values on the BACK input screen are correct:

- Press $<C$ trl $Z>$ to save the values and exit from the BACK input screen.

- Enier spectrum header information, if necessary.

The BACK program will calculate the ambient background radiation and print the results. 


\subsection{Perform General Spectrum Analysis}

Four Ge spectral analysis routines are available for which a specialized report is not required:

1. GAP is used where an upper limit on the area of each peak is limited to the $95 \%$ confidence level. Ambient background values are subtracted using the preanalysis technique (see Section 3.4.1).

2. GNUL has no upper limits, and the calculated photopeaks and their associated uncertainties are not restricted. The difference between GAP and GNUL is apparent only when the area of a peak is small in relation to its uncertainty. Ambient background values are subtracted using the preanalysis technique (see Section 3.4.2).

3. CBAT uses the same peak fitting algorithms as GNUL, but uses the concurrent ambient background analysis technique. This program allows you to select up to 22 different background spectra to be used in the analysis (see Section 3.4.4).

4. CBAG is identical to CBAT except that the three most recent backgrounds for the detector system are automatically selected (see Section 3.4.3).

All of the analysis routines are very flexible and permit you to select many different options. Figure 3-9 describes the functions performed by all four routines.

$\begin{array}{lc}\text { Function } & \text { Selectable } \\ \text { Produce output results in different types of activity units } & \text { YES } \\ \text { Produce output results in different quantity units } & \text { YES } \\ \text { Select isotope libraries } & \text { YES } \\ \text { Calculate input spectrum header values } & \text { YES } \\ \text { Automatically find peaks in spectrum } & \text { NO } \\ \text { Perform non-linear least squares fit of gaussians to peaks } & \text { NO } \\ \text { Determine values for selected isotopic lines (found or not) } & \text { YES } \\ \text { Assign isotopic identification to fit lines } & \text { NO } \\ \text { Subtract activity due to ambient background } & \text { YES } \\ \text { Do line interference decontamination } & \text { NO } \\ \text { Perform Parent-Daughter corrections } & \text { YES } \\ \text { Determine net concentration values (line value averaging) } & \text { NO } \\ \text { Print results of each peak fit and an isotope summary report } & \text { YES }\end{array}$

Figure 3-9. Functions performed by the general spectrum analysis routines. 
All of the Ge spectral analysis routines produce a printed output report that has three parts: peak description, isotope summary, and final summary. The peak description and the isotope summary are printed for all types of Ge anaiysis. The appearance of the final summary report depends on the type of sample from which the spectrum was collected.

The peak description outpui shows the results obtained from the fitting process (centroids, widths, etc.) and the associated nuclide lines obtained from the isotope library. A maximum of three lines can be associated with each peak in the spectrum, provided they are within $1 \mathrm{keV}$ of the energy of the peak.

The isotopic summary output groups together all the identified lines for a particular radionuclide and identifies the lines used to compute the final activity concentration value. This final concentration value can be the resu't of several different operations. The routine will print a flag number with each line to help you identify which operations were performed. A description of each of these flags is given in Appendix $\mathrm{E}$.

Sections 3.4.1 through 3.4.4 describe each of the general spectrum analysis routines.

3.4.1 Analyze Spectra Using GAP. Use the GAP routine if an upper limit on the area of each peak is to be limited to the $95 \%$ confidence level. Ambient background values are subtracted using the preanalysis technique.

'To run the GAP nutine:

- Type 'GAP' and press <cr>.

The GAP routine will dispiay the GAP input screen (see the example shown in Figure 3-10).

\begin{tabular}{|ll|}
\hline SPECTRUM SLOT (e.g., Al): & AI \\
PRINT SUMMARY REPORT ONLY? & NO \\
NUMBER OF SUMMARY REPORT COPIES: & 1 \\
ACTIVITY UNITS (pCi, uCi, dps, Bq, dpm): & uCi \\
PERFORM BACKGROUND CORRECTION? & YES \\
PRINT I.ESS-THAN VALUES? & NO \\
ISOTOPE LIBRARY: & MASTER \\
REQUIRED ENERGY LIBRARY: & ZEROLIM \\
ENTER SPECIRUM HEADER PARAMETERS? & YES \\
INTERFERENCE LIBRARY: & NOINTLIB \\
PERFORM PARENT-DAUGHTER CORRECTION? & NO \\
ELIMINATE EXCESSIVE DECAY TAGS? & YES \\
\hline
\end{tabular}

Figure 3-10. Example of the GAP input screen. 
If you want to change any of the default values:

- Press $<\mathrm{cr}>$ to move the cursor to the value you want to change.

- Enter the new value, which will appear to the right of the current value. For each menu item, only a few values are valid:

- $\quad$ SPECTRUM SLOT-the name of the spectrum file (e.g., A1).

- $\quad$ PRINT SUMMARY REPORT' ONLY?--either 'YES' or 'NO'.

If you enter 'YES', only the summary sheet will be printed.

- $\quad$ NUMBER OF SUMMARY REPORT COPIES - a positive integer greater than or equal to zero.

If you enter zero, GAP will not print a final summary page, so you will not need to edit the summary report.

- $\quad$ ACTIVITY UNITS - one of the following units: pCi, uCi, dps, Bq, or dpm.

The conversion to the selected activity will be printed on the final summary report. Activity units for the isotope summary list will be in $\mathrm{uCi}$.

- PERFORM BACKGROUND CORRECTION?-either 'YES' or 'NO'.

If you enter 'YES', then the concentrations of the background radionuclides that were last identified for this detector slot will be subtracted from the same radionuclide concentrations in the sample spectrum. If you enter ' $\mathrm{NO}^{\prime}$ ', then GAP will not perform the background subtraction function.

- $\quad$ PRINTT LESS-THAN VALUES?--also either 'YES' or 'NO'.

If you enter 'YES', then GAP will print the values for all radionuclides that have concentrations that have upper-limit concentration values.

- ISOTOPE LIBRARY - the name of the isotope library to use in the anc 'ysis.

The GE\$ISOTOPE \$LIB directory contains the isotope libraries.

- REQUIRED ENERCY LIBRARY - the name of the required energy library to use in the analysis.

The required energy library contains a list of radionuclide line energies for which the analysis code will be required to determine values. The GE\$LIMIT\$LIB directory contains the required energy libraries.

- ENTER SPECTRUM HEADER PARAMETERS?-either 'YES' or 'NO'.

If you enter 'YES', the HEAD program will run automatically after you exit the GAP input screen (see Section 3.2). 
- INTERFERENCE LIBRARY---the name of the interference library to use in the analysis.

Use this to clean up peaks containing counts from more than one isotope. The default 'NOINTLIB' will disable this feature of the analysis. The GE\$INTERF\$LIB directory contains the interference libraries.

- PERFORM PARENT-DAUGHTER CORRECTION?--either 'YES' or 'NO'.

If you enter 'YES', GAP will perform a parent-daughter correction according to the information contained in the parent-daughter library in the directory pointed to by the logical GE\$PD\$LIB. Also, GAP will print a separate 'Parent-Daughter' summary sheet, which will indicate the actions performed on the nuclides contained in the parent-daughter library (see Section 3.5).

- ELIMINATE EXCESSIVE DECAY TAGS?-either 'YES' or 'NO'.

If you enter 'YES', then isotopes requiring excessive decay corrections will have their tags removed from the peaks. Excessive decay correction is defined to be a correction in excess of 15 half-lives.

- Press $<c r>$ to replace the old value with the new one.

- Press <cr $>$ again to move the cursor to the next value you want to change. (If the cursor is at the bottom of the screen, press $<\mathrm{cr}>$ to move it to the top.)

When all the values on the GAP input screen are correct:

- $\quad$ Press $<$ Ctrl $Z>$ to save the values.

- Enter spectrum header information, if necessary.

If you wanted at least one copy of the summary report, the GAP routine will dispiay the summary report editing screen (see Figure 3-11 for an example)

$\begin{array}{cccc}\text { LINE } & \text { RADIONUCLIDE } & \text { SAMPLE ACTIVITY }(\mathrm{UCi} / \mathrm{CC}) & \text { ERROR } \\ \text { 1. } & \text { AR } 41 & 8.321 \mathrm{E}-03 & 2.80 \\ \text { 2. } & M N 54 & 1.409 \mathrm{E}-03 & 8.03 \\ \text { 3. } & \mathrm{MN} 56 & 3.362 \mathrm{E}-03 & 5.08 \\ \text { Do you wish to delete a line? If so, enter the line number. } & \\ \text { To edit the analysis results file, type 'E' } & \\ \text { To end editing, type 'Ctrl } Z \text { '. There are 0 ines left to display. }\end{array}$

Figure 3-11. Summary report editing screen.

By using the functions on the editing screen, you may delete radionuclides and see which radionuclides will be printed on the summary report. These features let you see which nuclides are present and which nuclides are valid without having to print the complete analysis results. Also, you can edit the summary results without having to reanalyze spectra. 
If you want to delete a radionuclide:

- Enter the number of the line you want to delete.

If you want to use the edit function:

- Type ' $E$ '. The EDT text editor will be invoked to allow you to examine the analysis output.

- $\quad$ Press $<\mathrm{Ctrl} Z>$ to end editing and redisplay the summary output results.

- Press <cr to display the next set of lines to edit.

When there are no more lines to display, the GAP routine will exit from the summary report editing screen and print the analysis results.

3.4.2 Analyze Spectra Using GNUL. The GNUL program has no upper limits imposed, so all photopeaks are printed regardless of the size of the uncertainty term. The difference between GAP and GNUL is apparent only when the area of a peak is small in relation to its uncertainty. Ambient background values are subtracted using the preanalysis technique.

To run the GNUL routine:

- Type 'GNUL' and press <cr .

- Enter the same values as for the GAP routine explained in Section 3.4.1.

- $\quad$ Press $<C$ trl $Z>$ to save the values.

- Enter spectrum header information, if necessary.

- Edit the summary xeport, if desired (see Figure 3-11).

The GNUL routine will then print the reports.

3.4.3 Analyze Spectra Using CBAG. The CBAG routine uses the concurrent background correction technique. The three most recent background spectra for the detector system are automatically selected. If there are less than three background spectra in the BKG\$SLOT directory for that detector system, the CBAG routine will use only the number of spectra that are available. The CBAG routine prints the background spectra photopeak results as part of the peak fit summary page.

To use the CBAG routine:

- Enter 'CBAG' and press <cr>.

- Enter the same values as for the GAP routine explained in Section 3.4.1.

- $\quad$ Press $<\mathrm{Ctrl} \mathrm{Z}>$ to save the values.

- Enter spectrum header information, if necessary.

- Edit the summary report, if desired (see Figure 3-11). 
3.4.3.1 Spectrum Analysis Using HP, a Derlvative of CBAG. The HP routine is identical to the CBAG routine, except that the HP routine does not quantify the activities of the radionuclides identified. Also, the HP input screen is identical to the CBAG input screen, except that the default values for the isotope, required energy, and interference library fields are specific to HP. Thus, the summary report lists only the radionuclides, with no quantification of their activities. Use the HP routine if the sample geometry is irregular and, hence, no efficiency table is available, making meaningful quantification impossible. (Such an instance would be when a health physics technician needs to know only which radionuclides might be present on a pipe wrench, hence the name HP.)

To use the HP routine:

- Enter 'HP' and press <cr>.

- Enter the same values as for the GAP routine explained in Section 3.4.1.

- $\quad$ Press $<$ Ctrl $\mathrm{Z}>$ to save the values.

- Enter spectrum header information, if necessary. (Make sure that efficiency table 2 is specified in the header information.)

- Edit the summary report, if desired (see Figure 3-11),

3.4.3.2 Spectrum Analysls Using SCREEN, a Derlvative of CBAG. The SCREEN input screen is identical to the CBAG input screen, except that the default values for the isotope, required energy, and interference library fields are specific to SCREEN, and the default activity units are in nanoCuries (nCi).

The SCREEN routine is based on the reporting requirements of the Department of Transportation. SCREEN does not print nuclides found in nature (naturals) in the summary report, and does not include either natural or required radionuclides in the editing list. Also, SCREEN calculates and prints total activities for those listed radionuclides that have true-positive activities.

If the grand total activity is above the specified limit of $2 \mathrm{nCi} / \mathrm{gram}$, the SCREEN routine prints a warning message as part of the surnmary report. Separate totals and their corresponding uncertainties are presented for the required radionuclides and for the other tagged, non-natural radionuclides. The uncertainties are propagated in the standard fashion for the uncertainty of a sum: the uncertainty of the total equals the square root of the sum of the squares of the individual uncertaintics.

To use the SCREEN routine:

- Enter 'SCREEN' and press <cr>.

- Enter the same values as for the GAP routine explained in Section 3.4.1.

- $\quad$ Press $<$ Ctrl $Z>$ to save the values.

- Enter spectrum neader information, if necessary.

- Edit the summary report, if desired (see Figure 3-11).

3.4.4 Analyze Spectra Using CBAT. Like CBAG, the CBAT routine uses the same concurrent background correction technique, but you are not limited to three background spectra. You may enter up to 22 background spectra you wish to use. 
To use the CBAT routine:

- Enter 'CBAT' and press <cr>.

- Enter the same values as for the GAP routine explained in Section 3.4.1.

- $\quad$ Press $<\mathrm{Ctrl} \mathrm{Z}>$ to save the values.

The CBAT routine will then display the background spectra selection menu, as shown in Figure 3-12.
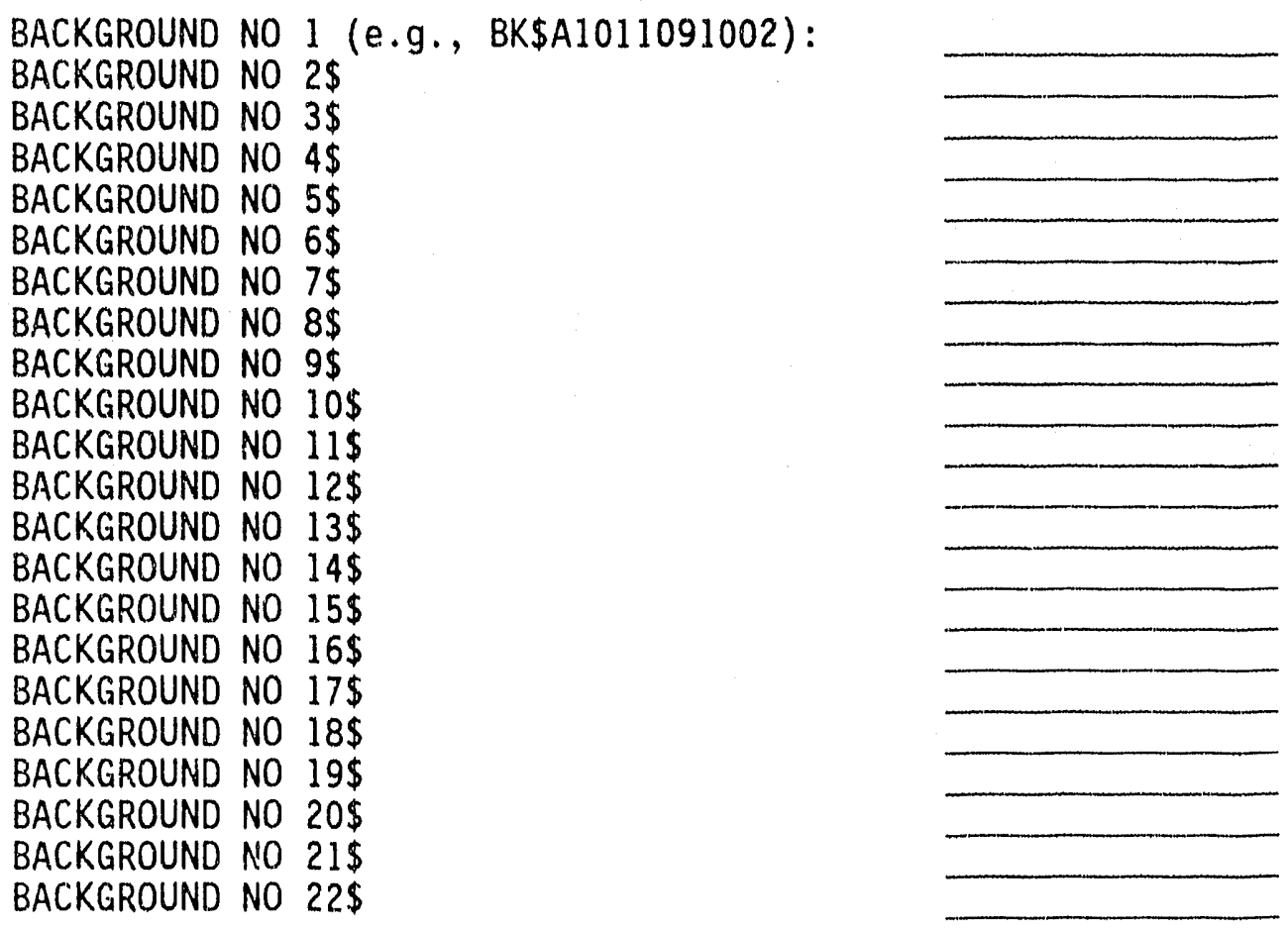

Figure 3-12. Background spectra selection menu.

- Enter spectrum IDs for up to 22 background spectrum files.

- Each entry must be in the form XX\$XnMMDDYYSSS (e.g., BK\$A1062492002), where 'XX' represents the save area directory in which the spectrum file is located (see Figure 3-7), and the remaining characters represent the name of the spectrum file (the extension. DGE is not necessary).

- $\quad$ Press $<\mathrm{Ctrl} Z>$ to start the spectrum analysis.

\subsection{Construct Parent-Daughter Files}

In any of the general analysis routines (GAP, GNUL, etc.), if you enter 'YES' for parent-daughter correction, VAXGAP will attempt to correct the activities measured for all nuclides listed in a parent-daughter file for their growth during the decay period and counting interval. 
Careful checks are required to identify daughters and granddaughters that might be in equilibrium with a longer-lived immediate precursor. When decay product activities are near equilibrium, the correlation between measured daughter activity and the daughter activity at the beginning of the decay interval is lost. If a daughter (granddaughter) is in equilibrium with its precursor, the daughter (granddaughter) activity is corrected for simple decay using the precursor half-life, and this will be noted in the parent-daughter summary that will be printed when the analysis finishes. The parent-daughter file is in the directory pointed to by the logical GE\$PD\$LIB and defines the nuclides to be corrected and their decay relationship.

When VAXGAP performs the parent-daughter correction, VAXGAP will read the PD_LIBRARY.LIB file (see Figure 3-13 for an example).

$$
\begin{aligned}
& \text { C COMMENTS CAN BE PUT IN FILE IF COLUMN } 1=C \\
& C \\
& 2,36,88, K R, 37,88, R B, 1.0 \\
& 3,53,133, I, 54,133, X E M, 54,133, X E, 0.0288 \\
& 2,54,138, X E, 55,138, C S, 1.0
\end{aligned}
$$

Flgure 3-13. Example of the parent-daughter library file.

- Enter decay chain descriptions in the format shown below.

Begin each decay chain description in column 1 . Separate each entry with a comma. Use only one line per decay chain for up to three niember decay chains.

nm,gpz,gpa,gpid,pz,pa,pid,dz,da,did,branch

where:

$$
\begin{array}{ll}
\mathrm{nm} & =\text { number of entries in chain (2 or 3) } \\
\mathrm{gpz} & =\text { grandparent nuclide } \mathrm{Z} \\
\mathrm{gpa} & =\text { grandparent nuclide } \mathrm{A} \\
\text { gpid } & =\text { grandparent chemical symbol (e.g., KR) } \\
\mathrm{pz} & =\text { parent nuclide } \mathrm{Z} \\
\mathrm{pa} & =\text { parent nuclide } \mathrm{A} \\
\mathrm{pid} & =\text { parent chemical symbol } \\
\mathrm{dz} & =\text { daughter nuclide } \mathrm{Z} \\
\mathrm{da} & =\text { daughter nuclide } \mathrm{A} \\
\text { did } & =\text { daughter chemical symbol } \\
\text { branch } & =\text { decay branching factor. }
\end{array}
$$

- Save the new PD_LIBRARY.LIB file. 


\section{OPERATING MISCELLANEOUS PROGRAMS}

You may use many small utility programs to manipulate spectral data files and their contents. Use EFF to maintain efficiency tables, use MAKE_LIB to create VAXGAP isotope libraries, and use SPECTRA to read, write, and move spectrum files. VAXPLOT displays and prints spectrum plots. Use EPB and ECB to edit a spectrum parameter block and a spectrum slot calibration file, respectively. PRHEAD displays and prints spectrum headers. Sections 4.1 through 4.7 describe these utility programs.

\subsection{Create and Modify Efficiency Curves/Tables (EFF)}

The efficiency tables used by the analysis routines are in the directory pointed to by the logical EFFICIENCY\$TABLES. The tables are normally referenced by a four-digit table number (e.g., 1154), but the file names actually consist of a two-character slot number, a four digit distance in centimeters, and the four-digit table number (e.g., A100101154). The slot number is used as a validity check to reduce operator errors when entering the efficiency number. The distance is also a validity check, but it allows you to create a group of tables for different distances and save the group as one table number.

Each table consists of up to 250 pairs of energy and efficiency values. There are no restrictions on the spacing between energies of the points, but the interpolation routine will produce the best results if more points are used in regions of the curve where the slope is changing the most. A typical curve consists of about 50 pairs of values with about half the points below $300 \mathrm{keV}$. Also, typical RML efficiency calibration curves span an energy range from 50 to $3000 \mathrm{keV}$.

To use the programs that maintain the efficiency tables:

- Enter 'EFF' and press <cr>.

VAXGAP will display the efficiency table maintenance menu, as shown in Figure 4-1.

$$
\begin{aligned}
& \text { Efficiency Table Maintenance Menu } \\
& 1 \text { - Set Print Destination } \\
& 2 \text { - Analyze Reference Standard Spectrum } \\
& 3 \text { - Generate Table of Experimental Results } \\
& 4 \text { - Fit Curve on windowed terminal } \\
& 5 \text { - Edit/Create Tables Interactively } \\
& 6 \text { - Display/Print Contents of Tables } \\
& 7 \text { - Plot Efficiency Tables (Log Plot) } \\
& 8 \text { - Plot Efficiency Tables (Linear Plot) } \\
& 9 \text { - Plot Efficiency Tables (EFF ENG**..A) } \\
& 10 \text { - Print Sorted List of Tables } \\
& 11 \text { - Test Interpolation of Tables } \\
& 12 \text { - Edit/Create Reference Standard Data Sheet } \\
& 0 \text { - Exit } \\
& \text { Enter Your Choice }
\end{aligned}
$$

Figure 4-1. Efficiency table maintenance menu.

- Enter the number of the action you want to perform. 
- When you have finished, enter ' 0 ' to exit from the menu.

Sections 4.1.1 through 4.1.12 explain each of the options in the efficiency table maintenance menu, respectively.

4.1.1 Set Print Destlnation. This option lets you select the printer for output. It is the same as the SETPRINTER command procedure. After you select ' 1 ' in the efficiency table maintenance menu:

- Select the name of the default printer.

If you select 'LASER', VAXGAP will ask you for the number of columns:

- If necessary, enter ' 80 ', '96', or ' 132 ' for the number of columns to print with the laser printer, then press $<\mathrm{cr}>$.

You can print reports using 80 columns, except when you analyze a reference standard spectrum.

4.1.2 Analyze Reference Standard Spectrum. Use this option to analyze a calibration source spectrum and store the calculated efficiencies in the EFFCRV\$RESULTS directory. This option can be run as a standalone program by typing the 'ECA' command at the DCL prompt. VAXGAP will display the efficiency curve analysis screen, as shown in Figure 4-2.

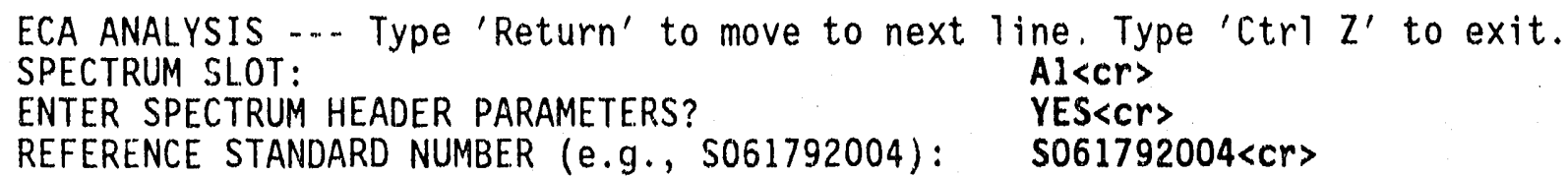

Figure 4-2. Efficiency curve analysis screen.

- Enter the spectrum slot (e.g., B3) that contains the calibration source spectrum to be analyzed; then press $<\mathrm{cr}>$.

- Enter either 'YES' or 'NO' for spectrum header parameters and press <cr>.

If this is a new spectrum, enter 'YES'. This will cause the 'HEAD' program to be run so you can enter header information. If you have already entered header information, enter 'NO'.

- Enter the ten-character calibration source VAX ID number for the sample that was counted and press <cr>.

The program will then analyze the spectrum and save the calculated efficiencies in the directory pointed to by the logical EFFCRV\$RESULTS. Result files will have the same names as the spectra they are produced from (e.g., A2062592015). VAXGAP will print a report showing the peak fits and the micro-curie results.

4.1.3 Generate Table of Experimental Results. Use this option to perform a weighted average of the saved results for up to 10 spectrum analyses.

- Enter the efficiency table name where the results will be saved. 
If experimental data already exist in the table, VAXGAP will ask if you want to continue:

- $\quad$ Enter ' $\mathrm{Y}$ ' or ' $\mathrm{N}$ ', if necessary, and press <cr>.

- Enter the result files to be used.

Result files have the same name as the spectra they are produced from (e.g., A2062592015). Each time the program asks for a result file, enter the next file to be included in the averages.

- When you have finished entering result files, enter a blank name to terminate data entry.

VAXGAP will calculate the averages and place them in the experimental data area of an efficiency table file. VAXGAP will also print a report that shows the input data, the results of the averaging, and the statistics of the averaging.

4.1.4 Flt Curve on X-WIndow TermInal or VAX Workstation. This option is the Efficiency Curve Fit (ECF) program, which lets you fit polynomial curves to experimental efficiency data and build a new efficiency table. This option is available only on an X-window terminal (see Section 5.1 for instructions).

4.1.5 Edlt/Create Tables Interactively. Use this option when new tables are created or when editing existing tables. The detector system ID, VAX ID number, detector number, source-to-detector distance, and title must be entered.

4.1.6 Display/Print Contents of Tables. This option lets you display and print the information in a particular efficiency table.

- Enter the number of the efficiency table and press <cr $>$.

- Enter ' $\mathrm{Y}$ ' and press $<\mathrm{c}$ - $>$ if you want the table to be printed. (The table will always be displayed on your terminal.)

4.1.7 Plot Efflclency Tables (Log Plot). Use this option to plot the contents of an efficiency table on the laser printer using $\log / \log$ scaling. The program will plot the actual values in the table as small circles. It will then call the VAXGAP efficiency lookup subroutine EFF and plot the values it returns for a range of points between the high and low value in the table. As an option, you may plot the experimental data as activityrelated error bars with small triangles at each end.

4.1.8 Plot Efflclency Tables (LInear Plot). Use this option to plot the contents of the low energy region (low view) of an efficiency taule on the laser printer using linear scaling.

- $\quad$ Enter the energy range (in $\mathrm{keV}$ ) to be plotted (e.g., 30,400).

The energy range should be two real numbers separated by a comma.

The program will plot the actual values in the table as small circles. It will then call the VAXGAP efficiency lookup subroutine EFF and plot the values it returns for a set of points in the specified range. As an option, you may plot the experimental data as activity-related error bars with small triangles at each end.

4.1.9 Plot Efflclency Tables (EFF`ENG**-A). Use this option to plot the contents of the high energy region (high view) of an efficiency table on the laser printer using linear scaling. The ' $A$ ' coefficient is calculated using energies of 400 and $1600 \mathrm{keV}$ as endpoints of a line with a slope of zero for the function $\mathrm{Y}=$ $\mathrm{EFF}^{*}\left(\mathrm{ENG}^{* *}{ }_{-} \mathrm{A}\right)$. This amplifies the discontinuities in the curve. 
- Enter the energy range (in $\mathrm{keV}$ ) to be plotted (e.g., 200,3000).

The energy range should be two real numbers separated by a comma.

The program will plot the actual values in the table as small circles. It will then call the VAXGAP efficiency lookup subroutine EFF and plot the values it returns for a set of points in the specified range. As an option, you may plot the experimental data as activity-related error bars with small triangles at each end.

4.1.10 PrInt Sorted Llst of Tables. With this option, you may use four different sequences (detector system, number, title, or detector) to sort all the efficiency tables available on the system and print them. Each type of report has the same contents and format.

4.1.11 Test Interpolation of Tables. Use this option to produce a numeric test of the VAXGAP efficiency lookup subroutine EFF.

- If you want to print the results, enter ' $Y$ ' and press <cr>. (The results will automatically be displayed on your terminal.)

- Enter the low, high, and step values, and press $<\mathrm{cr}$.

Separate each value with a comma (e.g., 200.0,205.0,0.1).

- Enter 'EFF' and press <cr>.

VAXGAP will generate a set of values between the low and high values, in the increment you entered for the step.

- Enter more sets of low, high, and step values for the program to process.

- When you have finished, press $<\mathrm{Ctrl} Z>$ to exit back to the menu.

4.1.12 Edit/Create Reference Standard Data Sheet. Use this option to maintain reference standard (calibration source) data sheets (VAX files) that are needed to calculate efficiencies. Each data sheet contains information about the energies, activities, and associated uncertainties that are present in a particular calibration source. These are stored in binary form in files located in the directory pointed to by the logical GAMMA\$STD.

You may create new files, change existing files, or print files. The program creates a temporary ASCII file of the data sheet and then uses the text editor to allow you to make changes. When you have finished making changes with the text editor, the ASCII file is read and converted to binary form for storage. Print data sheets by sending the ASCII file to the current default printer.

\subsection{Create VAXGAP Isotope Libraries (MAKE_LIB)}

This section lets you know how to create VAXGAP isotope libraries with the MAKE_LIB and NIL programs. First, you will create three ASCII files (parent-daughter, extraction, and add-delete) (see Sections 4.2.1 through 4.2.3). Then, when you run the MAKE_LIB program (see Section 4.2.4), it will use these files to extract selected radionuclide information from the radionuclide data base, which has a documented pedigree and contains referenceable information on radionuclide gamma-ray and X-ray decay data used by the RML. After the MAKE_LIB program has finished, it will produce a new library file, which you may edit. Finally, you will run the NIL program (see Section 4.2.5), which will process the new library file and create 
the final VAXGAP isotope library. The MAKE-LIB program produces another output file, which is named RGH.LOG and contains information about what processes were performed when MAKE-LIB extracted radionuclides from the radionuclide data base (see Section 4.2.6). Section 4.2.7 lists some items that are important to remember when creating a new VAXGAP radionuclide isotope library.

4.2.1 Create the Parent-Daughter Flle. This file contains information concerning the construction of parent-daughter radionuclides. Figure 4-3 is an example of a parent-daughter file. After you enter lines for the parent and daughter, they will be combined into a new radionuclide, a parent-daughter combination, which has the parent half-life. In the first parent-daughter combination in Figure 4-3, a new radionuclide called KRRB is created from a parent of $36 \mathrm{Kr} 88$ and a daughter of $37 \mathrm{Rb} 88$.

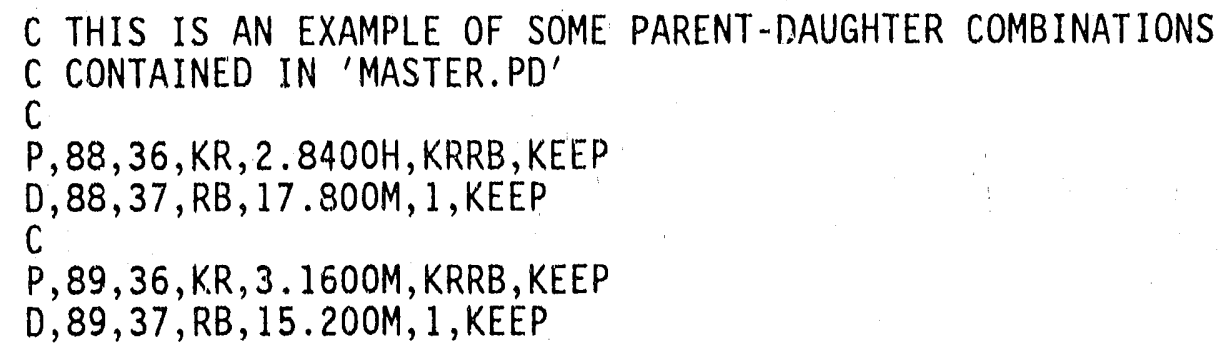

Figure 4-3. Example of a parent-daughter ASCII file.

- $\quad$ Enter 'EDT' and press <cr>.

Begin all entries in column 1 . You may enter comments by entering ' $C$ ' in column 1 , followed by the comment. Separate all field values with a comma.

- Enter a parent line in the form P,A,Z,ID,HL,NEW-ID,KEY.

$P$ is for parent; $A, Z$, and ID are the atomic number, the atomic weight, and the chemical symbol of the radionuclide; HL is the half-life as a unit of time (S, M, H, D, or Y); NEW-ID (e.g., KRRB) is the name of the new radionuclide; and KEY is either KEEP or DELETE.

The half-life must match the half-life value in the RGH data base and may have the form $1.456 \mathrm{E}+10 \mathrm{Y}, 145 \mathrm{~S}, 280.3 \mathrm{D}$, etc.

The NEW-ID may be any combination of four characters, but should begin with an alphabetic character.

Use KEEP to save the parent and daugnter lines after the new parent-daughter combination is created. If you enter DELETE instead, the program will delete the line from the working data base.

- On the next line after the parent line, enter a daughter line in the form D,A,Z,ID,HL,BRMF,KEY.

$D$ is for daughter, $A, Z$, and ID are the atomic number, the atomic weight, and the chemical symbol of the radionuclide; HL is the half-life as a unit of time (S, M, H, D, or Y); BRMF (e.g., 1) is the branching ratio multiplication factor (the decay mode branch); and KEY is either KEEP or DELETE. 
The branching ratio of the daughter lines will be multiplied by the BRMF value when the daughter lines are combined with the parent lines to create the parent-daughter radionuclide.

- After you have entered all parnt-daughter combinations, save the parent-daughter ASCII file with an appropriate name (e.8., MASTER.PD).

4.2.2 Create the Extraction Flie. In the extraction file, the information you enter will specify which radionuclides the MAKE_LIB program will extract from the radionuclide data base. Figure 4-4 shows an example of an extraction file.

$$
\begin{aligned}
& 4,7, B E, 4.604300 E+06 S, 50.0,3,477.59299 \\
& 8,19,0,2.691000 E+01 S, 50.0,3,1357.00000 \\
& 9,20, F, 1.103000 E+01 S, 50.0,3,1633.602 \\
& 11,22, N A, 950.7999 D, 50.0,1,1274.542 \\
& 11,24, N A, 5.407200 E+04 S, 50.0,3,2753.96509 \\
& 12,27, M G, 5.670000 E+02 S, 50.0,3,843.76001
\end{aligned}
$$

Figure 4-4. Example of an extraction ASCII file.

- $\quad$ Enter 'EDT' and press <cr>.

Begin all entries in column 1. You may enter up to 1000 entries. Separate all field values with a comma.

- Enter extraction lines in the form Z,A,ID,HL,LOW-ENERGY,MAX-ICODE,ENERGY-IC.

$\mathrm{Z}, \mathrm{A}$, and ID are the atomic weight, the atomic number, and the chemical symbol of the radionuclide to extract; HL is the half-life as a unit of time (S, M, H, D, or Y); LOW-ENERGY is the low cutoff energy value (e.g., 50) below which a radionuclide line will not be entered into the new library; in the same manner, MAX-ICODE is the largest I-CODE value that will be entered into the library; and ENERGY-IC is the energy of the line that is selected to have the I-CODE 1 value.

Isotope $x$-ray lines have a maximum I-CODE value of 12 and a minimum value of 8 . If you want to include $x$-rays in the new library, the value for MAX-ICODE must be no smaller than 8.

If the radionuclide data set contains an I-CODE 1 line energy that is different than the value in ENERGY-IC, MAKE_LIB will replace the I-CODE 1 line with the value in ENERGY-IC and will give the entry from the radionuclide data base an I-CODE value of 2 . If no line in the data base matches the value in ENERGY-IC, MAKE_LIB will display an error message.

If the energy is less than $1000.0 \mathrm{keV}$, a match will occur if the energy value is within $0.4 \mathrm{keV}$. For ener'gy values greater than $1000.0 \mathrm{keV}$, a match will occur if the energy values are within $0.8 \mathrm{keV}$.

- After you have entered all extraction entries, save the extraction ASCII file with an appropriate name (e.g., MASTER.EXTRACT).

MAKE_LIB will not extract a line from the data base if its branching ratio is less than $1 \%$ (except for I-CODE 1 lines). A line with a branching ratio less than $1 \%$ can be extracted from the data base with the use of an 'add' entry in the add-delete file (see Section 4.2.3). 
4.2.3 Create the Add-Delete Flle. Use this file to specify which lines for a particular radionuclide you want the MAKE_LIB program to add or delete. Figure 4-5 shows an example of an add-delete file.

$$
\begin{aligned}
& \text { C LIBRARY ADD-DELETE FILE FOR MASTER LIBRARY } \\
& \text { C } 6 / 6 / 91 \\
& \text { D, } 27,58, C 0,70.86 D, 511.0 \\
& D, 29,64, C U, 12.701 H, 511.0 \\
& D, 30,65, Z N, 244.25999 D, 511.0 \\
& A, 38,91, S R, 3.4272 E+4 S, 1280.855 \\
& A, 38,91, S R, 3.4272 E+4 S, 1413.506 \\
& A, 39,94, Y, 1.122 E+3 S, 2140.600 \\
& A, 40,97, Z R, 6.084 E+4 S, 703.761 \\
& A, 40,97, Z R, 6.084 E+4 S, 1276.128
\end{aligned}
$$

Figure 4-5. Example of an add-delete ASCII file.

- $\quad$ Enter 'EDT' and press <cr>.

Begin all entries in column 1. You may enter comments by entering ' $C$ ' in column 1, followed by the comment. Separate all field values with a comma. You may enter up to 100 'add' entries and up to 100 'delete' entries.

- Enter add-delete lines in the form F,Z,A,ID,HL,E.

$F$ is the function, either ' $D$ ' for delete or ' $A$ ' for add; $Z, A$, and ID are the atomic weight, the atomic number, and the chemical symbol; $\mathrm{HL}$ is the half-life as a unit of time ( $\mathrm{S}, \mathrm{M}, \mathrm{H}, \mathrm{D}$, or $\mathrm{Y})$; and $E$ is the energy of the line to add or delete.

The half-life must be within $5 \%$ of the half-life value in the data base.

The energy must not deviate from the data base value by over $0.01 \mathrm{keV}$.

- After you have entered all add and delete entries, save the add-delete ASCII file with an appropriate name (e.g., MASTER.AD).

4.2.4 Run the MAKE_LIB Program. Before you run the MAKE_LIB program, make sure you have created all the files you need (e.g., parent-daughter, extraction, and add-delete) to extract the information you want from the radionuclide data base.

- Enter 'MAKE-LIB' and press <cr>.

The MAKE_LIB program will ask for the names of the files, as shown in Figure 4-6.

- Enter the name of the parent-daughter file, including the directory where the file is located (e.g., GE\$ISOTOPE\$LIB:MASTER.PD); then press <Cr>. If you do not need a parent-daughter file, press $<\mathrm{cr}>$.

If the parent-daughter file is in your default directory, you do not need to enter a directory name. 


\section{SMAKE-LIB<Cr> \\ !Start program execution}

\section{ENTER PARENT-DAUGHTER FILE NAME: GE\$ISOTOPE\$LIB:MASTER.PD<Cr> \\ ENTER NEW LIBRARY FILE NAME: MASTER.ASCII $1<C r>$ \\ ENTER LIBRARY EXTRACTION FILE NAME: GE\$ISOTOPE\$LIB:MASTER. EXTRACT<Cr> \\ ENTER ADD-DELETE FILE NAME: GE\$ISOTOPE\$LIB:MASTER.AD<Cr> \\ $\$$}

Figure 4-6. Running the MAKE_LIB program.

- Enter the name that the new library file will be called (e.g., master.ascii)

If you do not include a directory name, MAKE_LIB will create the new library file in your fault directory.

- Enter the name (and directory, if necessary) of the extraction file and press <cr ; just press <cr $>$ if you do not need an extraction file.

- Enter the add-delete file name and directory, and press <cr>; just press <cr $>$ if you do not need an add-delete file.

The MAKF_LIB program will read the entries in the extraction file and check those entries against the add-delete file entries to delete and add lines. Entries in the add-delete file will not be used if an entry for the selected radionuclide was not in the extraction file or if a half-life or energy match was not made. If any entries in the add-delete file were not used, MAKE-LIB will print a list of them at the conclusion of the program.

The MAKE_LIB program will save the resulting radionuclides (that were extracted from the radionuclide data base) in the new library file (e.g., MASTER.ASCIII). An example of this file is shown in Figure 4-7.

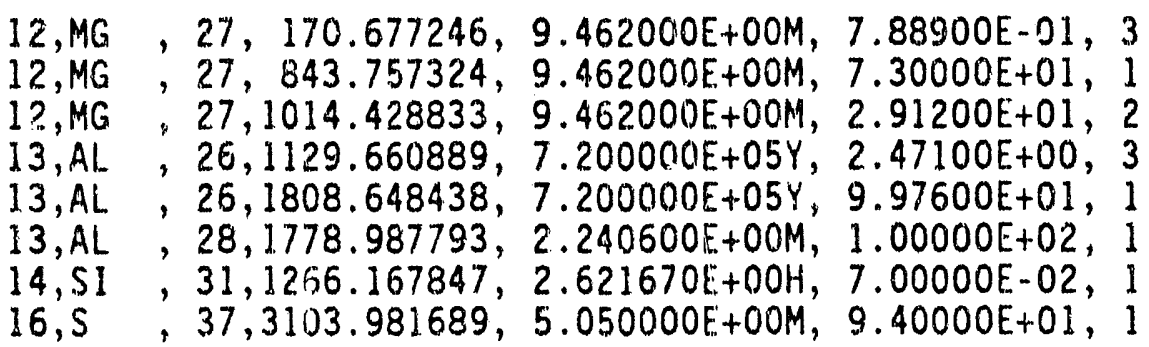

Figure 4-7. Example of the new library file.

Because the new library file is an ASCII file, you may delete or change lines; however, if you change values, the information will no longer be traceable to the radionuclide data base.

4.2.3 Run the N.L Program. Use the NIL program to construct a VAXGAP library from the new library file generated by the MAKE LIB program. Besides creating a VAXGAP library, the NIL prograrn performs three editing functions on the new library file: 
1. Checks all lines for identical energy values and adjusts those values by a small constant. VAXGAP does not permit identical energies for different nuclides.

2. Converts all half-life values to seconds.

3. Combines energy lines for a single radionuclide which are within $1 \mathrm{keV}$ into a single line.

The NIL program uses the following algorithm to adjust a set of identical line energy values $\mathrm{E}_{\mathrm{j}}$ :

$E_{i}=E_{i}+D$

where

$\mathrm{i}=$ from two to the number of identical lines for a given energy

$D=0.0001$ if $E_{i}$ is less than $1000 \mathrm{keV}$.

$D=0.001$ if $E_{i}$ is greater than $1000 \mathrm{keV}$.

$D$ is incremented by 0.0001 for each subsequent $i$.

The NIL program converts half-life values to seconds by using the conversion of $31,556,736$ seconds in a year (365.24 days in a year) for those radionuclides with a half-life greater than or equal to one year.

If two or more lines for a radionuclide are within $1 \mathrm{keV}$, VAXGAP will double-tag those lines with the same radionuclide identification. To prevent this from happening, the NIL program examines the set of lines for a radionuclide and for those lines within $1 \mathrm{keV}$, and combines them into one line. The NIL program uses we following algorithm to combine a set of lines with energy $E_{i}$ into one energy $E$ :

$E=\sum_{i=1} E_{i} * B R_{l} / B R T$

where

$$
\begin{aligned}
& B R_{i}=\text { branching ratio of line at energy } E_{i} \\
& B R T=\sum_{i} B R_{i} .
\end{aligned}
$$

The branching ratio of the new line at energy $\mathrm{E}$ is BRT.

To run the NIL program:

- Enter 'NIL' and press <cr>.

The NIL. program will ask for more information, as shown in Figure 4-8.

- Enter the name of the new library file that the MAKE_LIB program created (e.g., MASTER.ASCII) and press <cr>. If necessary, also enter the directory.

- Enier ' $N$ ' and press <cr>. 


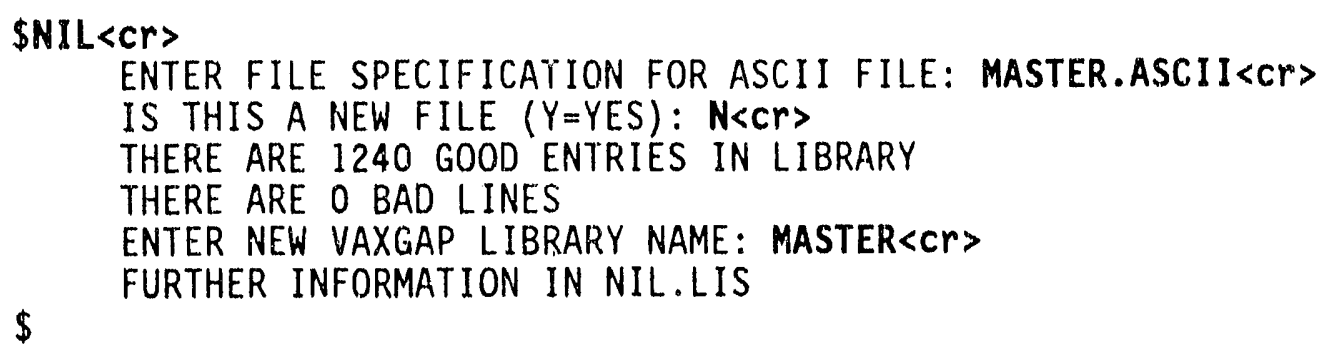

Flgure 4-8. Running the NIL program.

The NIL program will create a new VAXGAP library called MASTER.LIB and will store the new file in the GE\$ISOTOPE\$LIB directory.

Also, the NIL program will create a file called NIL.LIS, which contains information about duplicate energy values and energy combinations that were made. You may display the NIL.LIS file on your terminal or print the file.

4.2.6 Exam Ine the RGH.LOG Flle. Whenever you run the MAKE_LIB program, it will create an ASCII file called RGH.LOG, which will contain information on all the transactions made by the MAKE_LIB program. You may examine this file on your terminal with the EDT editor or print it.

Figure 4-9 shows an example of the type of entries that you might find in an RGH.LOG file.

The symbols '\%\%\%', '\$\$\$', '\#', and '!!!' each represent information from a certain type of routine in the MAKE_LIB program:

1. ' $\% \% \%$ ' is written by the routine that extracts the information out of the data base and loads it into computer memory. Its main function is to look for format errors in the data base and for other type of inconsistencies.

2. ' $\$ \$$ ' is written by the routine that processes the $x$-ray line information and determines the I-CODE values for the $\mathrm{x}$-ray lines only. The I-CODES for X-ray lines are stored in memory as negative numbers. When the X-ray lines are evaluated to determine whether to include them in the new library file, the sum of the absolute value of the (l-CODE) +6 is substituted as the $\mathrm{I}-\mathrm{CODE}$ value for the $\mathrm{X}$-ray lines. The maximum I-CODE value for the $\mathrm{X}$-ray lines is $\mathbf{- 2}$. 


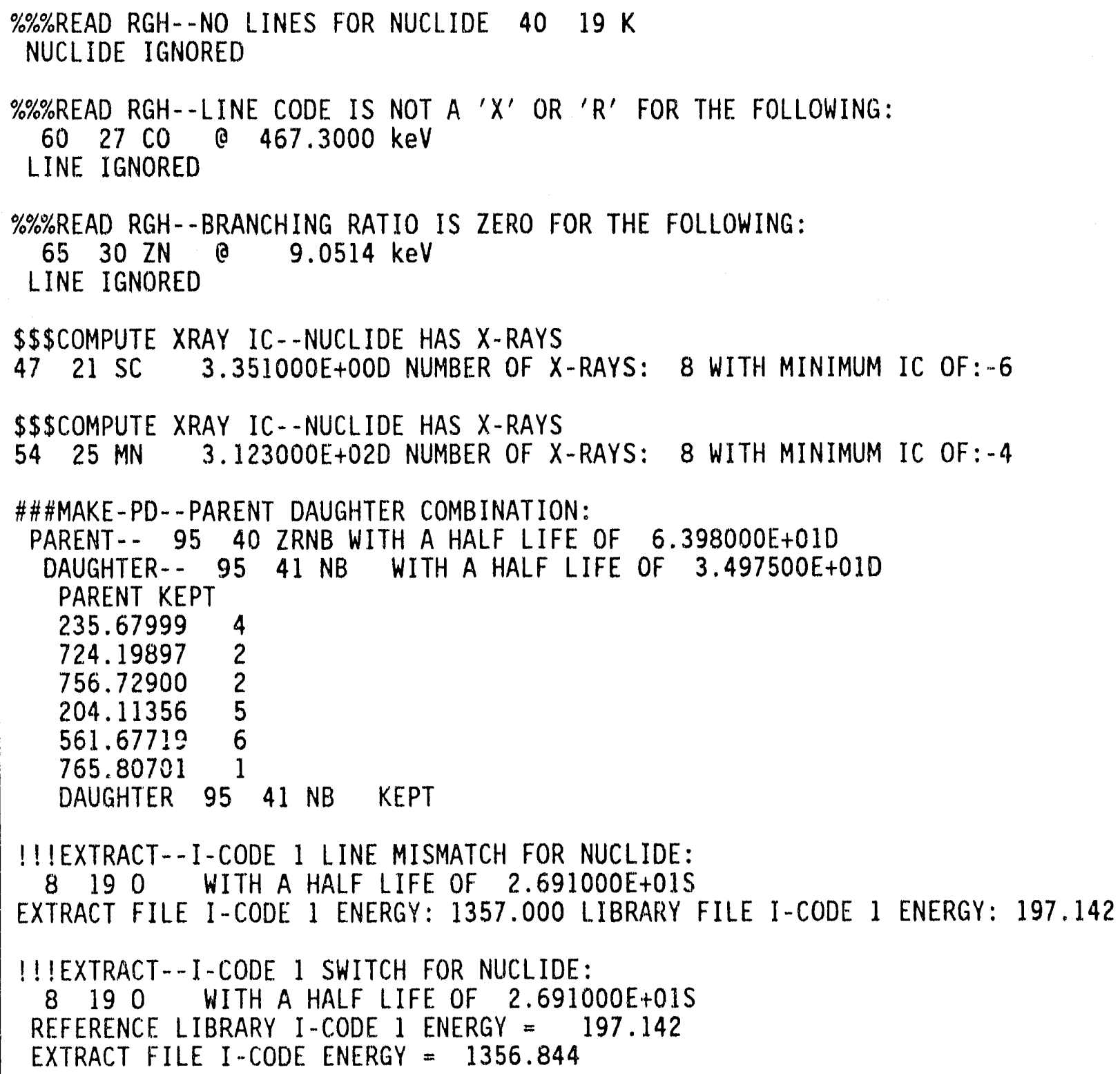

Figure 4-9. Example of entries in an RGH.LOG ASCII file.

3. ' ' is written by the routine that constructs the parent-daughter combinations from the parentdaughter file. A listing of all lines combined into the parent (and associated I-CODE values) is made. The I-CODE values are a recomputation of the all I-CODES based on the selected lines. The algorithm used to compute the new set of I-CODES is

$\mathrm{I} \cdot \mathrm{CODE}=1.501-\mathrm{F}+\mathrm{LOG}_{10}(\mathrm{MC} / \mathrm{BR})+0.5$

where

$$
\begin{aligned}
& \mathrm{MC}=\text { maximum branching ratio of all lines } \\
& \mathrm{BR}=\text { branching ratio of the line }
\end{aligned}
$$




$$
\begin{aligned}
& \mathrm{F}=0.0 \text { if (Max-energy - energy) is less thar } 500.0 \\
& \mathrm{~F}=0.5 \text { if (Max-energy - energy) is greater than } 500.0 \\
& \text { where } \\
& \qquad \begin{array}{l}
\text { Max-energy }=\text { the energy of the line with the maximum branching ratio } \\
\text { energy } \quad=\text { energy of the current line. }
\end{array}
\end{aligned}
$$

4. '!!!' is written by the extraction routine. Messages show all I-CODE switches and the entries in the extraction file for which none of the radionuclide lines met the extraction criteria.

4.2.7 Remember Important ltems. Keep the following items in mind when you create a new VAXGAP library from the data base:

1. With the EDT editor, you may edit all files used by the MAKE_LIB program. When you invoke the MAKE_LIB program and enter the name of each file, be sure to include the name of the directory where the files are located.

2. You may read information from the radionuclide data base, but you cannot make changes to it within VAXGAP.

3. You cannot edit the VAXGAP library with EDT. To examine information that is in a VAXGAP library, use the PRLIB program to print it.

4. Keep all input files (parent-daughter, extraction, etc.) in a secure location so that when changes and updates are made to the radionuclide data base, you can easily generate a revised VAXGAP library.

\subsection{Manipulate Spectrum Files (SPECTRA)}

The spectral manipulation program (SPECTRA) reads and writes spectrum files to magnetic tape, and moves spectrum files between save areas and slots.

To run the spectral manipulation program (SPECTRA):

- Type 'SPECTRA' and press <cr>.

SPECTRA will display the SPECTRA menu, as shown in Figure 4-10.

\section{SSPECTRA $\angle C r>$}

DO YOU WANT TO:

A) READ FROM TK-70

B) WRITE TO TK-70

C) TRANSFER SPECTRA FROM SAVE AREA TO SLOTSSPC

D) TRANSFER SPECTRA FROM DATA DISK AREA TO SLOT\$SPC

E) LIST SPECTRA IN SAVE AREA

F) LIST SPECTRA ON TK7O (NON-BACKUP)

G) RETRIEVE SPECTRA FROM A WEEKLY BACKUP TAPE

H) LIST ALL FILES ON A WEEKLY BACKUP TAPE

$-\rightarrow A<c r>$

2) QUIT

Figure 4-10. SPECTRA menu. 
- Enter the letter of the action you want to perform.

From all the options available with SPECTRA, only options A, B, C, and D are used routinely. The other options are installation-specific and not described here.

When you use magnetic tape:

- Load the tape on the computer.

SPECTRA will logically mount the tape and print the following messages:

\$MOUNT-I.WRITELOCK, VOLUME IS WRITE LOCKED

\$MOUNT-I-MOUNTED, MOUNTED ON_MUB6:

- When you are finished using the SPECTRA menu, enter ' $Z$ ' and press $<c r>$ to exit.

Sections 4.3.1 through 4.3.3 below explain how to perform options A through D, respectively.

4.3.1 Read from TK70 or TK50 Cartridge Tape. If you want to read spectrum files from the TK70 or TK50 magnetic tape, choose option A. SPECTRA will proceed with a series of questions and prompts for commands, which are shown in Figure 4-11.

DO YOU WANT A LIST OF SPECTRA PRINTED? N<cr

ENTER COMMAND ( $\angle C N T L>Z$ TO EXIT): READ $<C r>$

ENTER READ MASK (E.G., *, 052085*, 05*, 052385001): 062292003<cr>

ENTER DESTINATION DIRECTORY (BK, GN, ETC.)

- LEAVE BLANK TO USE DIRECTORY IN TAPE FILE: GN<Cr>

OUTPUT DATA TO DETECTOR SLOT $[Y / N]: Y\langle c r\rangle$

ENTER DETECTOR SLOT (E.G., A3): B2<cr>

CURRENT $=85072201$ DATA BLOCK \#19

Figure 4-11. Interface with SPECTRA for 'Read from TK70.'

SPECTRA will begin the 'Read from TK70' interface by asking if you want a list of printed spectra:

- $\quad$ Enter ' $\mathrm{Y}$ ' or ' $\mathrm{N}$ ' and then press $<\mathrm{cr}>$.

If you enter ' $Y$ ', you will receive a hardcopy listing of the spectrum files.

- Enter either the 'READ' or 'SKIP' command and press <cr>.

You may add a number to the end of the command to read or skip that number of spectra (e.g., READ6, SKIP3). 'SKIP' used alone is the same as 'SKIP1'.

'READ1' will read just one spectrum. If you enter just 'READ', SPECTRA will ask you to enter a read mask:

- Enter a read mask, if necessary, and press <cr>. 
You may enter a specific spectrum or use a wildcard $\left(^{*}\right)$ to select many spectra. For example, entering '061792002' tells SPECTRA to read only the second spectrum taken that day, '061792*' means to read all spectra gathered on $06 / 17 / 92$, and ' *' will read all remaining spectra on magnetic tape.

If you use any type of READ command, you will need to specify the destination directory where the spectra will be stored:

- Enter the destination directory, if necessary, and press <cr>.

You may specify a specific save area (e.g., BK, GN, BW-see Figure 3-7 for all the save area directory names) or you may use the directory written on the tape.

If you read a single spectrum (e.g., READ1, 061792002), SPECTRA will ask if you want to transfer the data to a detector slot:

- Enter either ' $\mathrm{Y}$ ' or ' $\mathrm{N}$ ', if necessary, and press $<\mathrm{cr}>$.

If you enter ' $N$ ', SPECTRA will store the spectrum in a save area directory.

If you enter ' $Y$ ', SPECTRA will ask you for the name of the detector slot:

- Enter the detector slot (e.g., A3), if necessary, and press $<\mathrm{cr}>$.

SPECTRA will transfer the data to the detector you specify.

SPECTRA will then display the status of the program or if a problem exists. As you skip spectra and read spectra from the tape, SPECTRA will display the current spectrum's ID and the data block being accessed. When you have finished reading spectra, the SPECTRA menu will again be displayed.

4.3.2 Write to TK70. If you want to write spectrum files to the TK70 magnetic tape, choose option B from the SPECTRA menu (Figure 4-10). SPECTRA will prompt you for answers or commands, as shown in Figure 4-12.

DO YOU WANT A IIST OF SPECTRA WRITTEN? N<Cr>

ENTER TAPE ID: TEST $\langle C P\rangle$

SKIP TO EOV $(Y / N)$ ? $Y\langle C r\rangle$

DATA ON TAPE WILL BE OVERWRITTEN - OK? (Y/N) Y<Cr>

ENTER SAVE DIRECTORY (E.G., 'GN'), OR ENTER 5-DIGIT ID: GN<Cr>

Figure 4-12. Interface with SPECTRA for 'Write to TK70.'

- Enter ' $Y$ ' if you want SPECTRA to print a list of spectra; then press <cr>.

- Enter the tape ID (e.g., TEST) and press <cr>.

SPECTRA will print the tape ID on all listings.

- Enter ' $Y$ ' if you want to skip to the end of the spectra already saved on the tape; then press <cr>. 
If you enter ' $Y$ ', SPECTRA will save all existing spectral data. If you enter ' $N$ ', SPECTRA will ask you to confirm your request to overwrite the data.

- Enter either ' $\mathrm{Y}$ ' or ' $\mathrm{N}$ ', if necessary, and press $<\mathrm{cr}>$.

If you enter ' $Y$ ', SPECTRA will begin writing data on the tape, so all existing information on the tape will be destroyed.

- Enter either the name of the save area (e.g., BW) or the five-digit ID number (e.g., 01002) and press $<\mathrm{cr}>$. (See Figure 3-7 for a list of the save area directories.)

SPECTRA will then display a menu for transferring spectra from the save area to the tape, as shown in Figure 4-13.

*** WRITE SPECTRA FROM 'GN\$SAVE' TO TAPE ***

DO YOU WANT TO WRITE: A) INDIVIDUAL SPECTRA

B) ALL SPECTRA IN GN\$SAVE

C) ALL SPECTRA TAKEN ON MMDDYY

D) ALL SPECTRA TAKEN BETWEEN MMDDYY AND MMDDYY

E) ALL SPECTRA TAKEN ON MCA $n$

F) ALL SPECTRA TAKEN ON MCA $n$ ADC $m$

G) SELECT SPECTRA WITH SEQ NO. XXXXX

H) CHOOSE NEW DEFAULT DIRECTORY

$-->D<c r\rangle$

Z) QUIT

Figure 4-13. Menu to write data to the tape.

The following list shows the information that SPECTRA will ask you for, depending on the letter you choose:

A. Enter the name of the data file (e.g., B2062492002.DGE) and press <cr>; if you are done, just press $<\mathrm{cr}>$.

B. No extra information needed.

C. Enter the date (e.g., 061792) and press $<\mathrm{cr}>$, or press just $<\mathrm{cr}>$ to abort the data transfer.

D. Enter the first and second date, pressing $<c r>$ after each date, or press just $<c r>$ to abort the data transfer.

E. Enter the letter of the MCA (e.g., B) and press <cr>, or press just <cr $>$ to abort the data transfer.

F. Enter the letter of the MCA and the number of the detector (e.g., B3) and press $<$ cr>, or press just $<c r\rangle$ to abort the data transfer.

G. Enter the five-digit ID of a save area (e.g., 01002) and press <cr $>$. Then, enter the first date (e.g., 061792) or '*' if you want all dates in the directory, and press <cr>. If necessary, enter 
the second date and press $<\mathrm{cr}>$. At any time, you may press just $<\mathrm{cr}>$ to abort the data transcer.

H. Enter either the name of a save area (e.g., BW) or the five-digit number (e.g., 01002) and press $<\mathrm{cr}>$.

- When you are finished transferring data, enter ' $Z$ ' and press $<c r>$ to exit from the menu (Figure 4-13).

Figure 4-10, the SPECTRA menu, will be displayed.

4.3.3 Transfer Spectra from Save Area to SLOT\$SPC. If you want to transfer spectrum files from \$SAVE save area directories to the SLOT\$SPC directory, choose option C from the SPECTRA menu (Figure 4-10). SPECTRA will ask you for the name of the detector slot and the file name of the spectrum in GN\$SAVE. See Figure 4-14 for an example.

ENTER DETECTOR SLOT (E.G., B3) : B3〈Cr>

ENTER SPECTRUM ID (E.G., B2072785009.DGE): B3081585001.DGE<Cr>

GN\$SAVE: B3081585001.DGE

SLOT\$SPC:B3.DAT

NUMBER OF DATA CHANNELS IS : 4096.000

Figure 4-14. Example of transferring spectra from a save area directory to the SLOT\$SPC directory.

- Enter the name of the slot (e.g., B1) and press $<\mathrm{cr}\rangle$.

- Enter the file name of the spectrum (e.g., B 1061792012.DGE) and press <cr .

The spectrum will then be transferred to the slot. The associated calibration file in the slot will not be altered. The SPECTRA menu will then be displayed. Option D performs a similar operation, but takes the spectrum from the appropriate \$DATA save area.

\subsection{Plot Spectra (VAXPLOT)}

Use the spectral plotting program (VAXPLOT) to display and print any spectrum stored on the VAX disk. These files can be located in either the SLOT\$SPC directory or one of the save area directories (e.g., GN\$SAVE).

- Enter 'PLOT' and press <cr>.

- Enter the name of the spectrum file (e.g., A1 or A1081792009) and press <cr $>$.

If you enter a two-character ID, VAXPLOT will retrieve the file from the SLOT\$SPC directory.

If you enter the full 11-character name, VAXPLOT will ask you to designate which save area the file is located in: 
- If necessary, enter either the two-character ID (e.g., BW) or the five-digit number (e.g., 01002) that corresponds to the save area directory and press <cr $>$. (See Figure 3-7 for the list of save area directories.)

VAXPLOT will display the VAXPLOT input screen, as shown in Figure 4-15.

If you want to shainge any of the default values:

- $\quad$ Press $<c r>$ to move the cursor to the value you want to change.

VAXPLOT -.. Type 'Return' to move to next line. Type 'Ctrl $Z$ ' to exit.

SPECTRUM SLOT OR ID: $\quad$ A1040188025

FIRST DECADE, NUMBER OF DECADES: AUTO

COUNT SCALE (LOG OR NORMAL): LOG

NUMBER OF PLOT PASSES: $\quad 2$

TYPE PLOT (POINT, ASTERISK, OR LINE): LINE

$X$-AXIS LABEL (CHANNEL OR ENERGY): ENERGY

REGION OF INTEREST (START, END): $\quad 0,3000$

PLOT DEVICE (LASER, LN05, VT): VT

Figure 4-15. Example of the VAXPLOT input screen.

- Enter the new value, which will appear to the right of the current value. The following list explains each field in the VAXPLOT input screen:

- FIRST DECADE, NUMBER OF DECADES - the first or baseline decade and the total number of decades you want.

Enter two integers separated by a comma (e.g., 1,4).

The first decade of the plot is that power of 10 from which the channel contents are subtracted before they are plotted. Values below this point are not plotted.

The number of decades is the power of 10 that is added to the first decade to determine the top value for the channel contents. Values above this point are not plotted.

Enter 'AUTO' if you want all the spectral data to be plotted. VAXPLOT will calculate the first decade and number of decades automatically.

- COUNT SCALE (LOG OR NORMAL)-controls whether the Y-axis will be a logarithmic scale or a linear scale. 
If you enter 'LOG', the logarithm (base 10) of the counts will be plotted. If you enter 'NORMAL', the actual counts will be plotted. The Y-axis will be scaled appropriately to match the data.

- NUMBER OF PLOT PASSES--an integer between 1 and 4.

This is the number of passes (complete plots across the width of the paper) you want the program to make. The total number of channels to be plotted is divided by this value to determine the number of channels plotted for each pass.

- TYPE PLOT (POINT, ASTERISK, OR LINE)-designates how each point will be plotted.

Enter 'POINT' if you want a small dot for each channel. 'ASTERISK' will produce an '*' for each channel. If you enter 'LINE', each channel in the spectrum will be connected with a line.

- X-AXIS LABEL (CHANNEL OR ENERGY)-designates how the counts in a channel will be plotted.

If you enter 'ENERGY', each plot pass will use a linear approximation for that particular segment of the spectrum.

- REGION OF INTEREST (START,END)-controls the portion of the spectrum to be plotted.

Enter two integers separated by a comma (e.g., 100,500).

If the $\mathrm{X}$-axis label is channel, enter the region of interest in the range from 0 to 8191 channels. If the $\mathrm{X}$-axis label is energy, enter the region of interest in the range from 0 to $3000 \mathrm{keV}$.

The region of interest is divided by the number of passes to determine how many channels will be plotted on each pass. This is adjusted to provide tic marks at even intervals.

- $\quad$ PLOT DEVICE (LASER, LN05, VT)-designates the output device for the plot.

LASER selects the LN03 laser printer; LN05 selects the DEClaser 2150; VT displays the plot on your terminal.

- Press $<c r>$ to replace the old value with the new one.

- Press $<\mathrm{cr}>$ again to move the cursor to the next value you want to change. (If the cursor is at the bottom of the screen, press $<$ cr $>$ to move it to the top.)

When all the values on the VAXPLOT input screen are correct:

- $\quad$ Press $<\mathrm{Ctrl} \mathrm{Z}>$ to save the values and create the plot.

If you display the plot on your terminal, press <cr $>$ when you have finished viewing the plot. You will then get a menu that allows you to adjust the plot parameters, display the plot again on your terminal, print that plot, or exit. 
If you print the plot, VAXPLOT will display a message on your terminal that the plot is completed and that it has been queued to the printer.

On the printed plot of the spectrum, in the top center of the plot just under the plot border, you will see the spectrum's alphanumeric header. Also, in the center of the plot below the top X-axis, you will see the 11-character spectrum ID number.

If either of these spectrum designators consists of jumbled or unreadable characters, it may mean that you plotted data that had not been processed through the HEAD program (see Section 3.2).

If data from a foreign system was used to plot a spectrum, the spectrum's alphanumeric header will be the 61 st through the 135th characters in the header record, terminated by a carriage return (13) or null (0). The spectrum ID number will be the first two characters of the file name plus the 12 th through the 20th characters in the header record.

\subsection{Edit the Spectrum Parameter Block (EPB)}

Each spectrum file contains a parameter block, which is a collection of information about that spectrum (see Appendix C). You may use the EPB program to modify the contents of the parameter block.

- $\quad$ Enter 'EPB' and press <cr>.

- Enter the name of the spectrum file (e.g., A1 or A1081792009) and press <cr>.

If you enter a two-character ID, EPB will retrieve the file from the SLOT\$SPC directory.

If you enter the full 11-character name, EPB will ask you to designate which save area the file is located in:

- If necessary, enter either the two-character ID (e.g., BW) or the five-digit number (e.g., 01002) that corresponds to the save area and press $<\mathrm{Cr}\rangle$. (See Figure 3-7 for the list of save area directories.)

EPB will then display the EPB Batch input screen, as shown in Figure 4-16.

- Press the 'arrow' keys to move the cursor until the parameter you want to modify is highlighted and press $<\mathrm{cr}>$.

EPB will display that parameter's value and ask you if you want to change it:

- If you want to change the value, enter the new value. If not, just press $<c r>$.

EPB will save the new value and display the EPB Batch input screen again.

- When you have finished modifying values, highlight 'EXIT' and press <cr>.

\subsection{Edit the Spectrum Slot Calibration File (ECB)}

You may change values in the calibration block of a spectrum slot. This would be necessary if the widths of photopeaks in the spectrum change substantially due to gain changes. 
E D I T PARAMETER BLOCK

Use arrow keys to select parameter,
then press "RETURN".

EXIT LTIME RTIME START_TIME START_DATE S_NUM COLIECT_TIME COLLECT_DATE COLLECT_HOUR SMPL_FRACTION EFF_MULT EFF_TABLE

EFF_FILE DISTANCE VOLUME VOLUME_UNITS NO_CHANNELS TOTAL

SYS_FLOW SYS_VOLUME SYS_VOLUME_UNITS POWER COLL_POS ERR_EFF

ERR_GEO ERR_OTH SC_SUM MCA_SYSTEM ADC_NUM ZERO_OFFSET EA_CO

EB_CO EC_CO WA_CO WB_CO SA_SUM SB_SUM NO_PULSER

PULSER_RATE PLS_ONE_KEV PLS_TWO_KEV PLS_CHANNELS PLS_ONE_START

PLS_TWO_START PLS_ONE_PS PLS_TWO_PS CAL_PULSER_WD LOW_PULSER_WD

Figure 4-16. Example of an EPB Batch input screer.

- Enter 'ECB' and press <cr>.

- Enter the two-character ID of the spectrum slot (e.g., A1) and press <cr>.

The ECB program will display all the calibration file entry parameters for that spectrum slot. Figure 4-17 is an example of a spectrum slot's calibration block.

Channel Zero: 1.5250E-01

Energy Linear Term: 3.6217E-01

Width Zero Term: $2.7864 \mathrm{E}+00$

Energy zero term: $-3.8246 E-02$

Energy Quadratic term: $-6.2544 E-09$

Width linear term: $7.0014 \mathrm{E}-06$

Error Matrix: 11 1.380E-03 2 1.4340E-09 $32.8274 \mathrm{E}-17$ 4 - $1.2911 \mathrm{E}-06$

$51.6113 E \cdots 10 \quad 6-1.8908 E-13$

Random Sum Correction Coeff. A: $1.0000 E+00$ B: 0.0 C: 0.0

Number of Pulsers: 2 Pulser rate: 50 Pulser Channels: 128

1st Pulser Start Channel: 7936 offset: 7281

2nd Pulser Start Channel: 8064 Offset: 969

$\langle$ Ctr] Z terminates data entry

PF1 Aborts program

PF2 Erases field

Figure 4-17. Example of a calibration block. 
- Using the 'arrow' keys, move the cursor to the parameter you want to modify.

- Press 'PF2' to erase the contents of the field.

- Enter the new value and press $<$ cr>.

- For the 'Number of Pulsers' parameter, enter either ' 2 ' or ' 0 ' and press <cr>.

For spectrometers equipped with EG\&G Idaho's precision pulser, the parameter 'Number of Pulsers' controls whether the pulser information is used for calibration of the spectrometer.

If you enter ' 0 ', then pulser data will not be used in the calibration.

If you enter ' 2 ', then the pulser data will be used for calibration, and you must then enter valid entries for the following parameters:

- Pulser rate-the number of pulses (per amplitude) injected per second (the normal rate is 50).

- Pulser Channels--the number of channels dedicated to each pulser amplitude. The total number of channels used for the pulser data will be twice the number entered for this value.

- 1 st Pulser Start Channel-the starting channel in the spectrum where the low amplitude pulser data begins.

- Offset (1st pulser) - the number of channels added to the low amplitude pulser to place the pulser data in the indicated spectrum region. This value is deternined at spectrometer setup time and is the value coded into the low amplitude offset switches in the pulser interface.

- 2nd Pulser Start Channel-the starting channel in the spectrum where the high amplitude pulser data begins.

- Offset (2nd pulser) - the number of channels added to the high amplitude pulser to place the pulser data in the indicated spectrum region. This value is determined in the same manner as the low amplitude offset.

- When you have finished changing values, press $\langle\mathrm{Ctrl} \mathrm{Z}\rangle$ to terminate data entry and exit the program.

\subsection{Print the Spectrum Header (PRHEAD)}

You may print the spectrum header and the informational parameters that are stored with the spectrum data.

- $\quad$ Enter 'PRHEAD' and press $<\mathrm{cr}>$.

- $\quad$ Enter the file name of the spectrum you want to print and press $<\mathrm{cr}>$.

- Enter ' $Y$ ' and press <cr> if you want the information printed on the line printer. PRHEAD will automatically display the header information on your terminal. 


\section{OPERATING MISCELLANEOUS PROGRAMS ON X-WINDOW WORKSTATIONS}

This section describes four graphics programs that perform different functions. Section 5.1 explains the Efficiency Curve Fit (ECF) program, which fits polynomial curves to experimental efficiency data and lets you build a new efficiency table.

In Section 5.2, you will learn how to run DAS, a program that displays in real time the spectral data being accumulated on a multi-channel analyzer. DAS also displays collected spectra that are stored in spectrum disk files (slots).

Another graphics program is GINA, which allows you to define the fit limits and background values for a non-linear fit of the data to a gaussian function and display the resulting function values (see Section 5.3).

The XTP program, explained in Section 5.4, performs spectrum analysis and displays the peak fit results.

Since these programs use the DEC Windows interface, you must run them on VAX workstations or $X$-window terminals. In this section, the notation 'click' means to press the left button on the mouse. If the middie or right button of the mouse should be clicked, the instructions will specify which button to click.

\subsection{Generate Efficiency Curves (ECF)}

Use the ECF program to fit polynomial curves to experimental efficiency data and to create and modify efficiency tables.

- Enter 'EFF' and press <cr.

The efficiency table maintenance menu will be displayed (see Figure 4-1 in Section 4.1 for an example).

- Type ' 4 ' to select 'Fit Curve on windowed terminal' (the ECF program).

- Enter the name of an efficiency table and press <cr $>$.

The table name must be 10 characters long (e.g., A400001111), and the table file must reside in the EFFICIENCY\$TABLES directory.

- Enter the range of the table and press <cr . If you want to keep the default values, just press <cr.

Separate the range values with a comma (e.g., 50.0,3000.0). The range values (in $\mathrm{keV}$ ) are the starting energy and the ending energy of the table, and may be between 40.0 and $4000.0 \mathrm{keV}$. These values are for display scaling purposes and to place a boundary around the generation of new table values.

The ECF program will display function boxes, a plot of the efficiency curve points (if there are any), the experimental data points, the region boundaries that have been defined, region boundary values (in $\mathrm{keV}$ ), the number of points in each region, the degree of polynomial used to fit the experimental points in that region, and a reduced chi-squared number that was computed when the fit was made (see Figure 5-1).

Sections 5.1.1 through 5.1.14 describe each of the function boxes. 


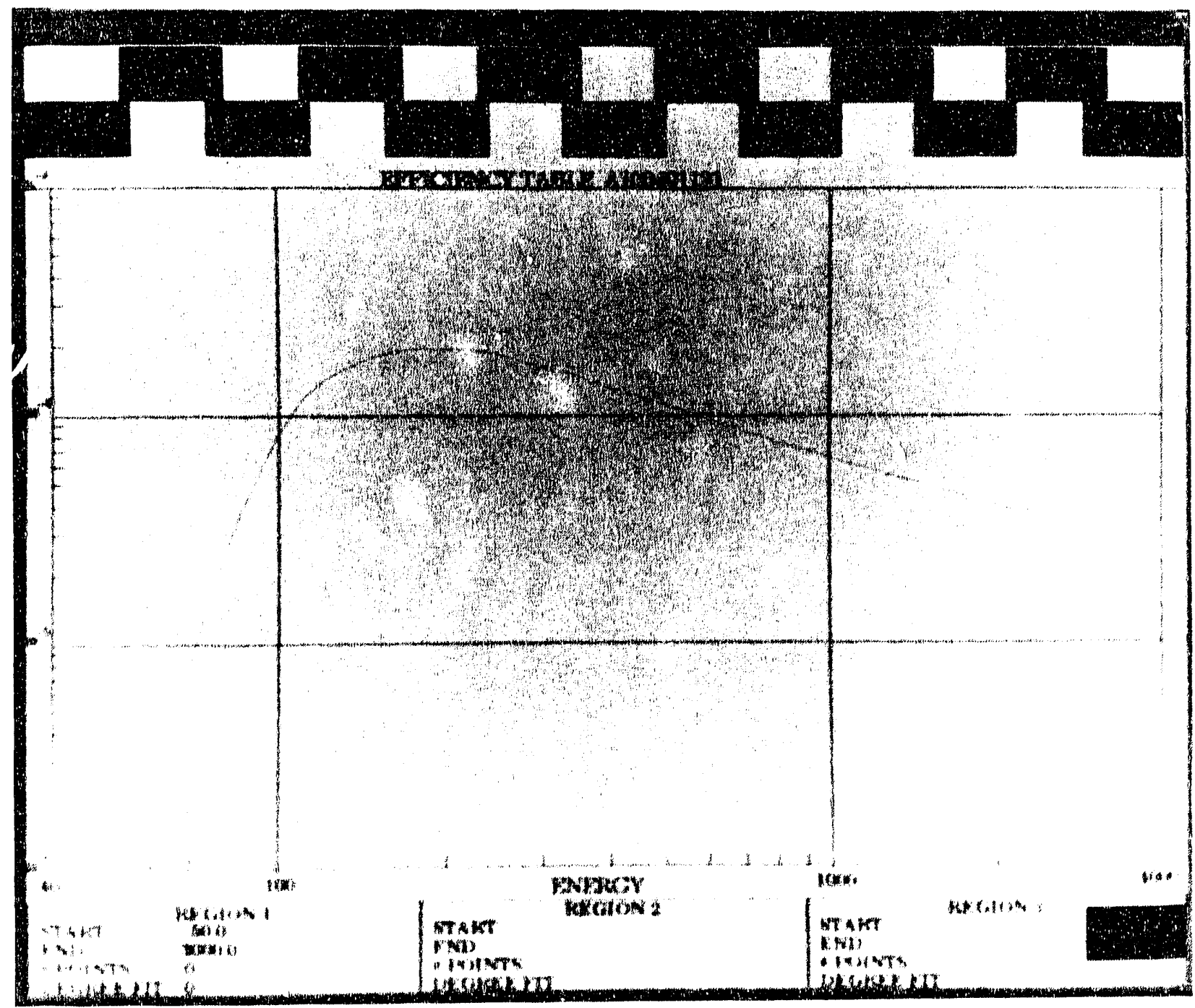

Flgure 5-1. ECF selection screen.

5.1.1 Add Exp Polnt. Use this function to add experimental data points when you need to extend the efficiency table beyond the energy range of experirnental data. Also, this function provides additional points in areas where the polynomial curve does not follow the desired shape.

- Click the ADD EXP POINT function box.

- In the graph display area, click wherever you want to place new points.

- When you have finished, click the DONE function box to quit.

The uncertainty for the selected points will be set to zero, so the added points will be shown as small black dots instead of vertical lines.

5.1.2 Delete Exp Polnt. Use this function to adjust the position of data points or to remove incorrect data points.

- Click the DELETE EXP POINT function box.

- Click the points you want to delete.

- Click the DONE function box to quit. 
5.1.3 Move Exp Polnt. You may move experimental data points that were added manually, but you cannot move experimentally measured points. Moving a point will change only the efficiency of the point; the energy remains the same as the original point.

- Click the MOVE EXP POINT function box.

- Move the desired points to new locations.

To move a point: Place the cursor on the point, hold down the left mouse button, move the point up or down to a new location, and release the mouse button.

- When you have finished moving points, click the DONE function box to quit.

5.1.4 Vlew LOG/LOG. If you click the VIEW LOG/LOG function box, ECF will show experimental points as vertical lines, indicating the uncertainty of each point. If a fit has been made, it will be shown as a curve through the points. ECF will show the high and low range of the table as dashed lines, as well as any region boundaries that have been defined. The region parameters will also be shown below the plot of the data.

5.1.5 View Low. Click the VIEW LOW function box to display a linear plot of the low energy portion of the efficiency table (below $400 \mathrm{keV}$ ). ECF will show experimental data, current table definitions, and a curve through the current definitions (see Figure 5-2).

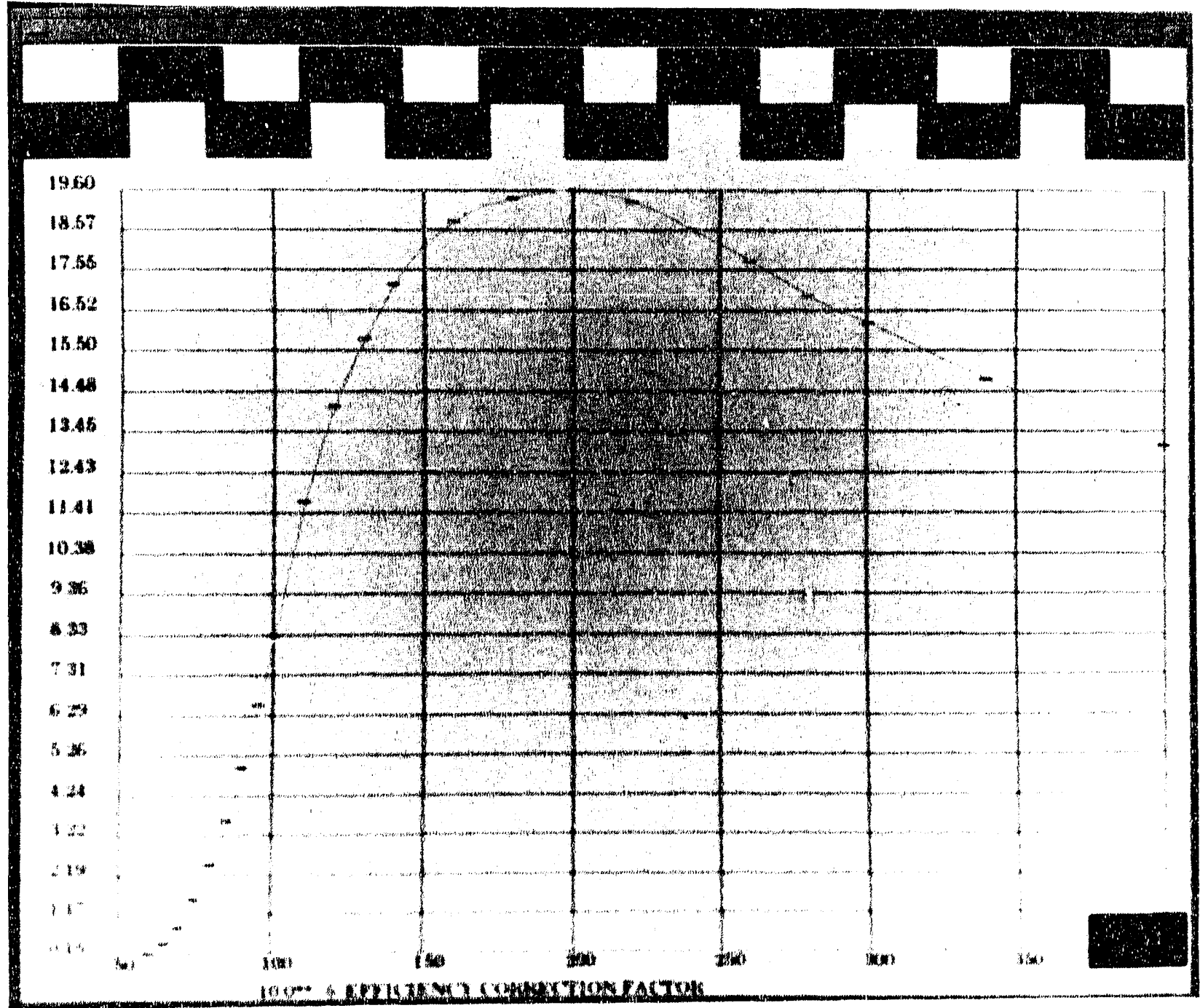

Figure 5-2. ECF yiew low screen. 
5.1.6 View HIgh. The VIEW HIGH function shows an 'energy $x$ efficiency' plot of the data above 200 $\mathrm{keV}$. The exponential term of this equation will initially be calculated from the data, but it can be selected manually to change the slope of the curve. Like the VIEW LOW function, VIEW HIGH will show experimental data, current table definitions, and a curve through the current definitions (see Figure 5-3).

5.1.7 Define Reglon. Use the DEFINE REGION function to define the number of regions that the curve will be divided into and the boundaries between the regions. This function is available only when the $\log / \log$ view is on the screen. Each region will have a separate polynomial fit to it.

- $\quad$ Click the DEFINE REGION function box.

- Select the number of regions you want to define $(1,2$, or 3$)$.

- Click on the graph where you want each region to end.

Intermediate boundaries will be adjusted to the nearest data point. Any data points outside the ends of the high and low region will not be used for the curve fit.

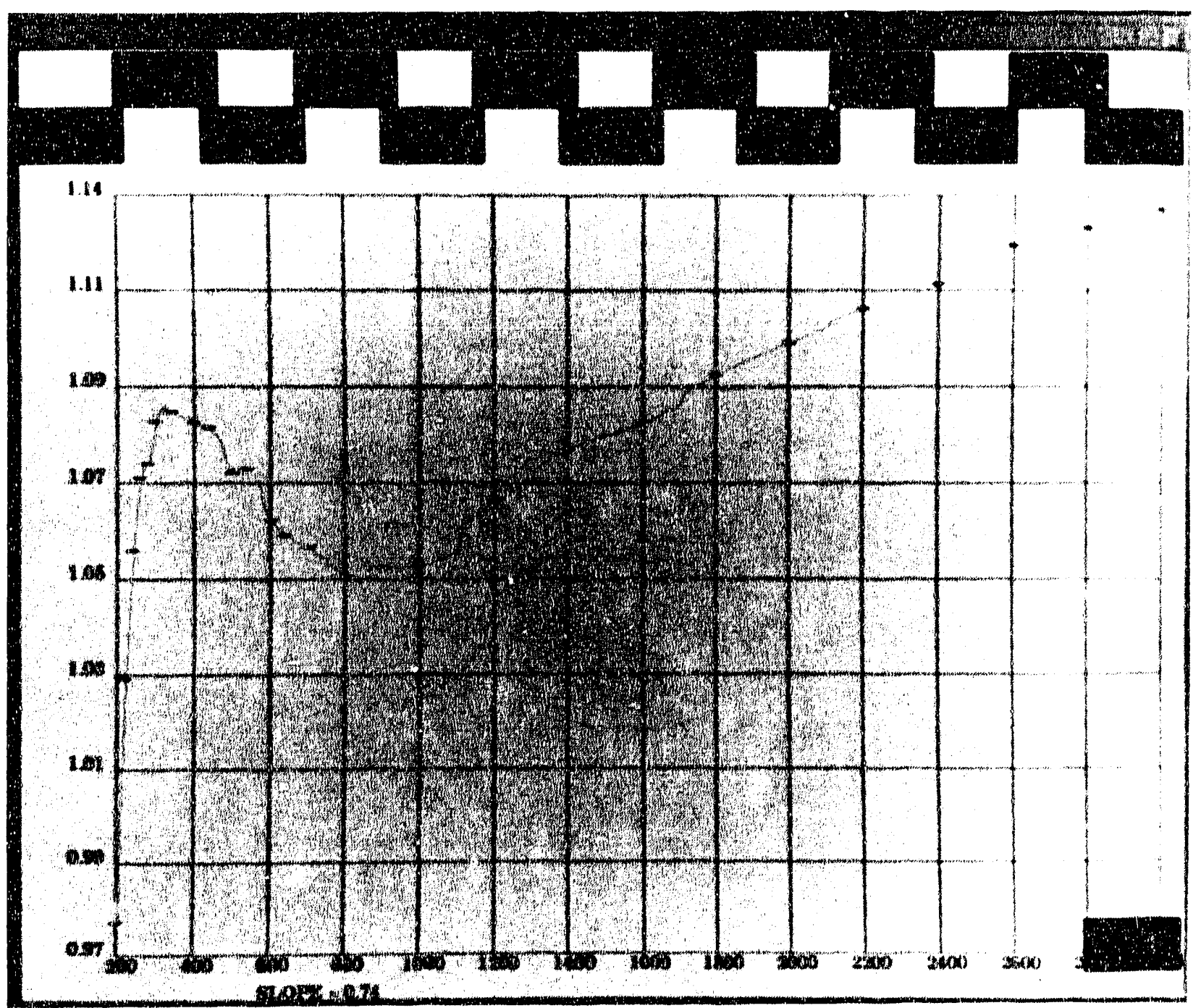

Figure 5-3. ECF view high screen. 
5.1.8 DefIne Degree. With the DEFINE DEGREE function, you may define the degree of the polynomial to be used for each region. This function is available only when the $\log / \log$ view is on the screen.

- Click the DEFINE DEGREE function box.

The ECF program will display the maximum degree permitted for the current number of data points. (There must be one more data point in the region than the degree of the polynomial.) Currently, the maximum degree of polynomial supported by VAXGAP is 10 .

- Enter polynomial degrees for each region:

- $\quad$ Click the digit boxes ( 0 through 9 ) to select the desired digits.

- If necessary, click ERASE to remove the last digit you selected.

- Click ENTER to save the polynomial degree for the region.

- If you want to terminate the DEFINE DEGREE function without saving any values, click EXIT.

5.1.9 Fit Curve. Use the FIT CURVE function to determine the coefficients of the polynomial to be used for each region of the curve. This function is available only when the $\log / \log$ view is on the screen.

As the number of data points and the degree of the polynomial increases, the computer will take longer to process the calculations. After ECF completes the fit, ECF will save the new set of points for the current efficiency table, but they will not be written to the disk until you exit the program.

5.1.10 Change Slope. Use this function to adjust the slope, a, of the "eff.energy-a" plot of the data. You may enter a value or let ECF calculate a value from the data. Small changes in this value have a large effect on the screen. A value of 0.85 is typical for most curves.

- $\quad$ Click the CHANGE SLOPE function box.

- Click either MANUAL or CALCULATED.

If you select CALCULATED, ECF will automatically compute the slope and then exit out of this function.

If you select MANUAL, you will need to enter a value:

- Click the digit boxes and the ' ' box to select a value.

- Click ENTER to save the value and exit.

5.1.11 Move FIt Polnt. The MOVE FIT POINT function adjusts the fit points in order to refine the shape of the efficiency table. This function should be used to smooth any "ringing" produced by the polynomial fit. Moving a point will change only the efficiency of the point; the energy' remains the same as the original point. This function is not available on the $\log / \log$ view because the resolution of the screen is not adequate for this function.

- Click the MOVE FIT POINT function box.

- Move the desired points up or down to new locations.

To move a point: place the cursor on the point, hold down the left mouse button, move the point to a new location, and release the mouse button.

- When you have finished moving points, click the DONE function box to quit. 
5.1.12 Deflne LIne. Use the DEFINE LINE function to draw a straight line on the screen, which is sometimes useful when you analyze and construct efficiency curves.

- Click the DEFINE LINE function box.

- Draw as many lines in the graph region as you desire.

To draw a line: Place the cursor where you want to begin the line, hold down the left mouse button, move the cursor to where you want to end the line, and release the mouse button.

To erase a line: Select one of the view functions (e.g., VIEW LOG/LOG, VIEW LOW, VIEW HIGH).

5.1.13 Adjust Table. With the ADJUST TABLE function, you may multiply the efficiency table values by a constant value within a selected energy range. The energy range can include the whole table or any fraction of it.

- Click the ADJUST TABLE function box.

- Select the energy range of the curve within which to make the adjustment.

To select the range, click on the curve at the beginning position, then click again at the end of the range.

- $\quad$ Select ' $Y$ ' if the energy values are correct.

If you select ' $N$ ', ECF will erase the region markers and prompt you to select new region values.

- Enter an adjustment factor, which will be applied at the starting and ending positions of the region.

From these two adjustment factors, the ECF program will then compute two coefficients, $S$ and $Z$, for a function, $f$, such that

$f($ energy $)=S *$ energy $+Z$,

$f($ region's start energy) $=$ the adjustment factor at the start of the region, and

$f$ (region's ending energy) = the adjustment factor at the end of the region.

Efficiency table values within the selected region are then multiplied by the value of the function, $f$ (energy), where energy is the corresponding energy of the efficiency value. The resulting efficiency curve will then be displayed, and the program will restart the process by asking you to select a new region.

- When you have finished, click QUIT to terminate the process.

5.1.14 Qult. Use the QUIT function to update the efficiency table and return to the efficiency table maintenance menu.

- $\quad$ Click the QUIT function box.

- Enter ' $Y$ ' and press <cr> to insert efficiency table values and experimental point information, and retum to the efficiency table maintenance menu.

If you enter anything but ' $Y$ ', ECF will not modify any of the table values and will return you to the efficiency table maintenance menu. 


\subsection{Display Spectra (DAS)}

The DAS program displays, in real time, spectra as they are collected from detectors. Also, you may display spectral data after they have been stored in the directory pointed to by the logical SLOT\$SPC.

In addition, DAS provides a first-look photopeak identification function, which finds the peaks in a spectrum and associates the peak energies with radionuclide identifications out of the master nuclide library.

- Enter 'DAS' and press <cr>.

The DAS program will display the DAS selection screen, as shown in Figure 5-4.

- Click either the MCA (detector system) or SLOT function box.

If you click MCA, the DAS program will retrieve the spectrum directly from the detector system.

If you click SLOT, DAS will retrieve the spectrum from the directory pointed to by the logical SLOT\$SPC.
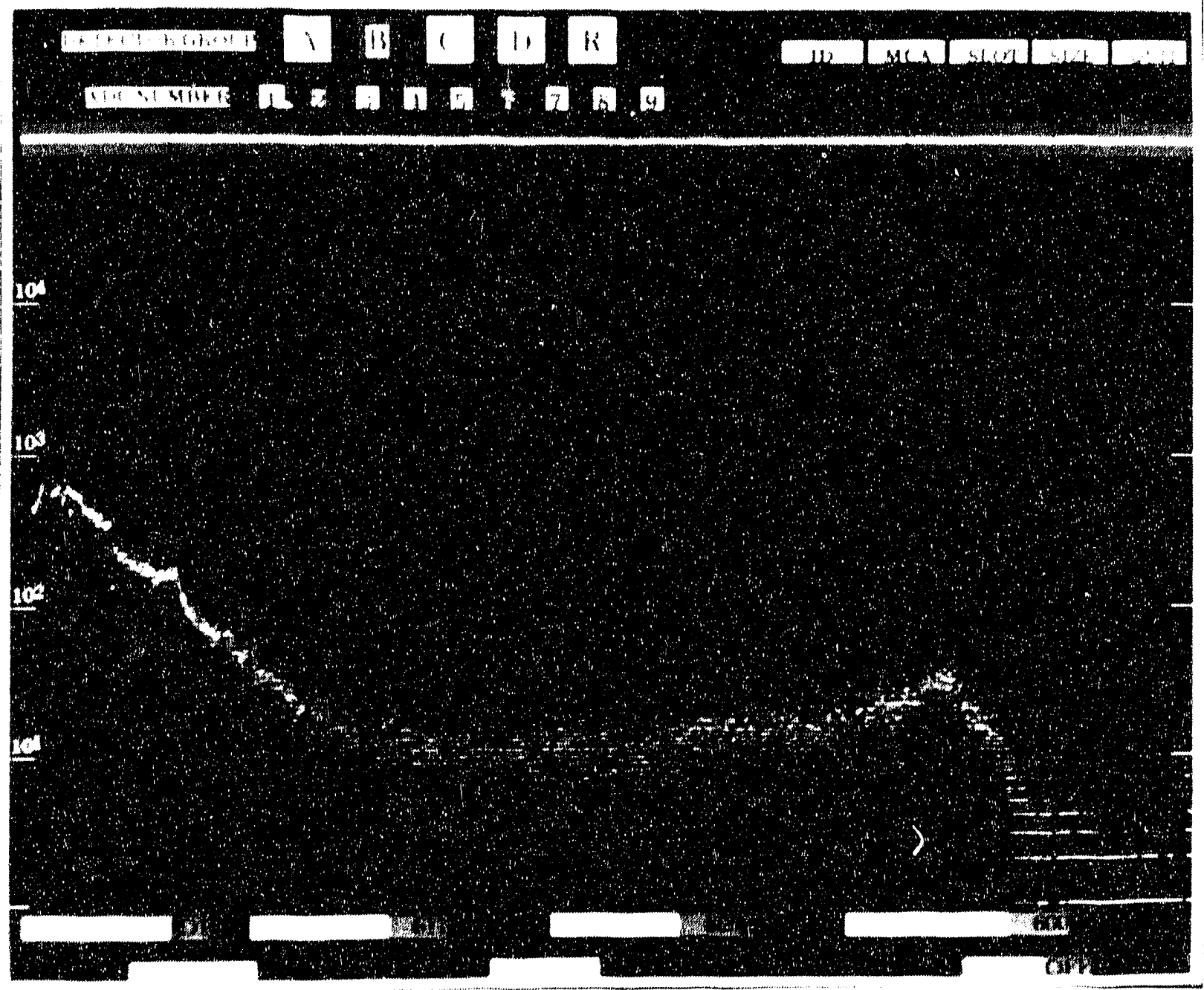

Figure 5-4. DAS selection screen. 
MCA is the default, which means that you do not need to click the MCA function box.

Select a detector group and an ADC number to tell DAS which spectrum to display (e.g., B3):

- Click a detector group box (A, B, C, D, or R).

The box you click determines which detector group you want to retrieve the spectrum from.

- Click an ADC number (1 through 9).

The ADC number is the specific number of the spectrum in that detector group.

The DAS program will retrieve the spectrum and display it on the screen. You can click in the spectrum display area to place a marker (a small vertical line) on a spectrum channel. DAS will display, in the status display area of the window (the bottom of the screen), the channel energy and nuclide identification associated with that energy.

5.2.1 Change the Slze of the Display. You may use the SIZE function to change the number of channels displayed in the window:

- $\quad$ Click the SIZE function box.

- Click the value for the number of channels you want to display (256 to $8 \mathrm{~K}$ ).

To automatically zoom in on 256 channels, move the cursor in the spectrum display area and click the middle mouse but!on. DAS will center the enlarged region of the spectrum at the position where you clicked.

5.2.2 Move the Starting Channel. The red bar graph shows how many channels are being displayed and where in the spectrum the displayed channels are located. The length of the bar is scaled to the number of channels displayed. For example, if the number of channels is $8 \mathrm{~K}$, then the bar is drawn completely across the window; if the number of channels is $4 \mathrm{~K}$, then the bar length is half the window width, etc.

The starting position of the bar indicates where the data display begins in the spectrum. You may move the starting channel of the spectrum display:

- In the green region where the red bar graph is located, move the cursor to the place you want the beginning channel of the spectrum to be displayed.

- $\quad$ Click the mouse to shift the beginning channel.

In the green region, the channel number values show where the spectrum display is positioned. In the spectrum display area, the bottom left number shows the beginning channel, and the right number shows the ending channel.

5.2.3 Identlfy Nuclides. You may use the ID function to identify the nuclides associated with prominent photopeaks in the spectrum. When you click the ID function box, the standard VAXGAP peak location subroutine finds the channel numbers of all peaks in the spectrum. After these channel numbers are converted to energy, this energy is used to tentatively identify the nuclide associated with each photopeak.

The DAS nuclide library is located in the GE\$ISOTOPE\$LIB directory. Initially, this library is a copy of the MASTER nuclide library. You can copy any VAXGAP library into DAS.LIB, and that library will be used for identification. 
- $\quad$ Click the ID function box.

- Examine the identification of each photopeak.

If the energy of any photopeak in the spectrum is not within $1 \mathrm{keV}$ of the photopeak energy of an entry in the nuclide library, DAS will display "NT" on that photopeak.

- Because the ID function does not determine a photopeak's exact centroid (center of mass only), the centroid and nuclide identification might be in error.

\subsection{Fit Peaks Interactively and Display Spectra (GINA)}

With the GINA program, you can interactively select background regions, fit limits, and peak centroids, and initiate a fit of the peak to a gaussian function. The results of the fit (centroids, widths, and quality-of-fit numbers) are displayed as a plot of the resulting gaussian function with the spectral peak data, which is useful in examining peak shape and resolution of peak doublets, and in determining background values.

If you plan to print a hardcopy of the resulting plot, you must define which laser printer you want to print the plot on by defining the que narne in GINA\$QUE:

- Enter the "Define" command and include the printer que you want to use (e.g., DEFINE GINA\$QUE LASER).

To begin the GINA program:

- Enter "GINA" and press <cr>.

- Enter the name of the spectrum file (e.g., A1 or A1081792009) and press <cr>.

If you enter a two-character ID, GINA will retrieve the file from the SLOT\$SPC directory.

If you enter the full 11-character name, GINA will ask you to designate which save area the file is located in:

- If necessary, enter the two-character ID (e.g., BW) that corresponds to the save area directory and press <cr>. (See Figure 3-7 for the list of save area directories.)

The GINA program will display the spectrum in the GINA selection screen (see Figure 5-5 for an example). At the bottom of the screen, the row of values preceded by the letter " $\mathrm{C}$ " represent the channel numbers, which are scaled across the screen. The second row (labeled E) indicate the energy of the channels displayed in the first row.

The following list explains the function boxes in the GINA selection screen:

ZOOM SIZE-determines the number of channels that will be displayed when you want to 'zoom' in on a specific spectral region. 


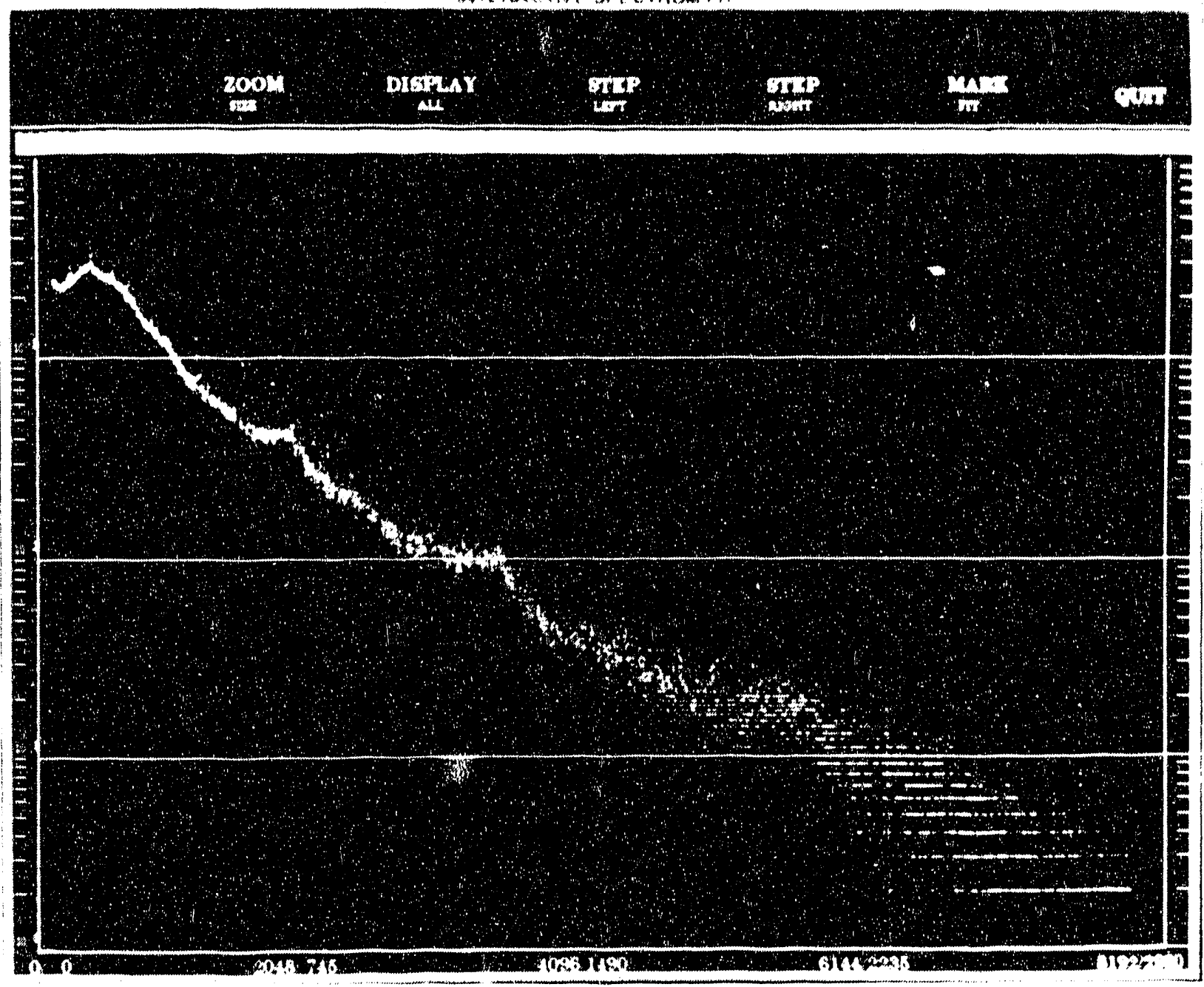

Figure 5-5. GINA selection screen.

- $\quad$ Click the ZOOM SIZE function box.

- Click the number of channels you want displayed $(128,256,512$, or 1024).

To zoom in on a region:

- Click the area you want to zoom in on.

If you are zoomed in on a region and you use the ZOOM SIZE function box to change the channel display, GINA will immediately resize the display.

DISPLAY ALL-displays the entire spectrum.

If you are zoomed in on a region:

- Click the DISPLAY ALL function box to resize the display to a full spectrum.

STEP LEFT-moves the spectrum display to the left the number of channels specified by the value in ZOOM SIZE. 
STEP RIGHT-moves the spectrum display to the right the number of channels specified by the value in ZOOM SIZE.

QUIT - terminates the execution of GINA and prints the results of the last fit if you saved the fit.

- When you are ready to quit, click the QUIT function box.

MARK FIT - lets you set background values, fit limits, and peak centroids, and initiate a fit of a peak.

- Click on a region to zoom in on it.

- $\quad$ Click the MARK FIT function box to display the MARK FIT screen, shown in Figure 5-6.

- $\quad$ Place background marks (i.e., '[' and ']') to designate regions that will be used as background values.

Click the left mouse button to place a left bracket. Then click again to place a right bracket. GINA will toggle between left and right brackets.

All channel values between brackets are averaged together to establish a background value for that set of brackets.

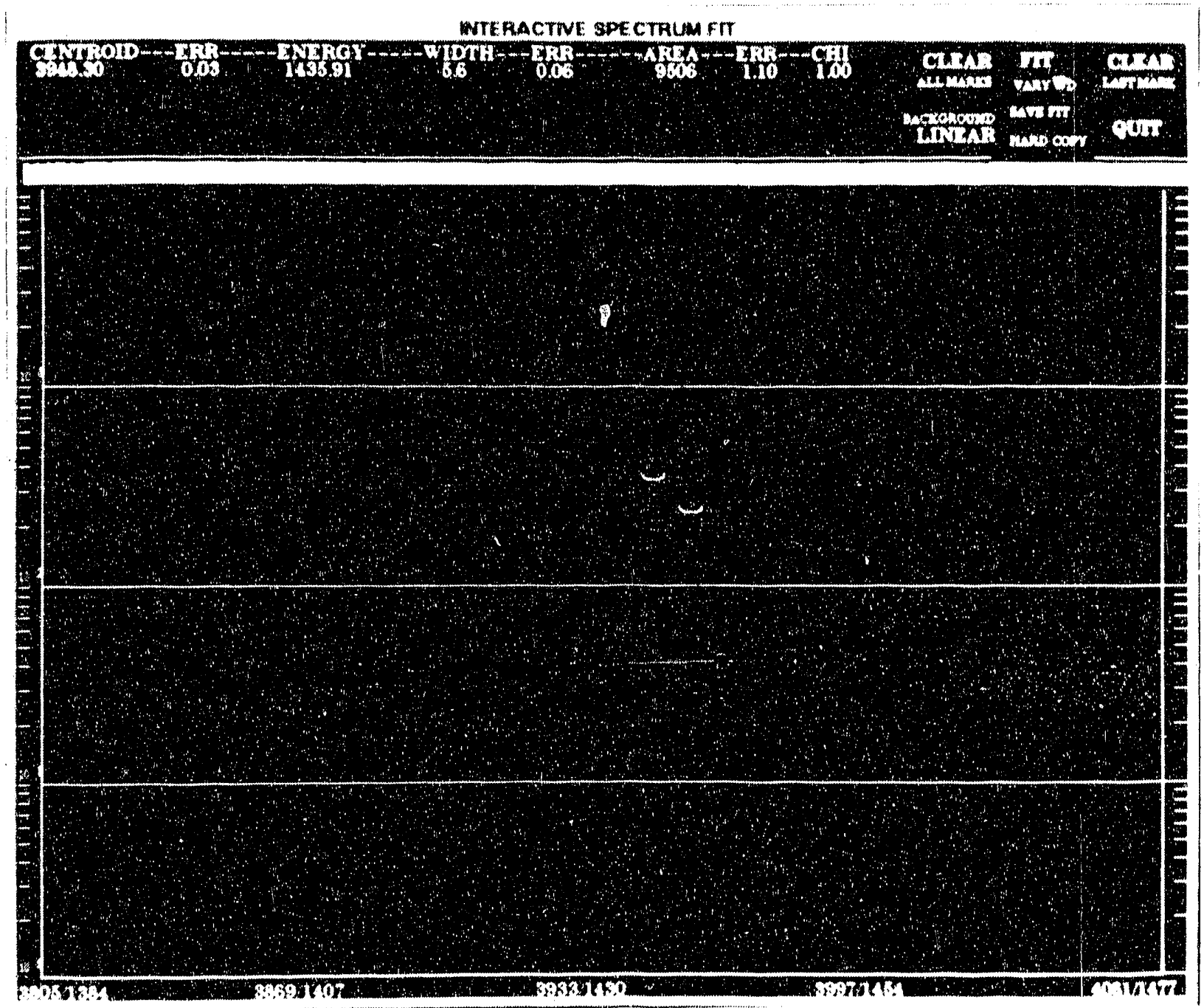

Figure 5-6. GINA mark fit screen. 
You must place two sets of background marks for each peak or set of peaks being fit.

- Place fit limit marks (i.e., ' $U$ ') to designate the left and right fit limits of a peak or set of peaks being fit.

Click the middle mouse button to place a fit limit mark on the starting channel of the fit region. Click the middle mouse button again on the ending channel. The fit region must contain at least three channels.

- $\quad$ Place peak centroid marks (i.e., '|') to identify the peak centroids to be used in the fit.

Click the right mouse button to place peak centroid marks. You must identify between one and five peaks for each fit. The centroid must be between the fit limits. The centroid mark identifies the initial centroid value for the fit. However, as the peak fitting is performed, this value will be adjusted to get the best peak fit.

The following list explains the function boxes in the MARK FIT screen:

CLEAR MARKS-deletes all fit marks and plots of the fit results.

CLEAR LAST MARK—-deletes only the last mark that was placed in the spectrum.

BACKGROUND LINEAR--determines the type of background that will be used to fit the peak. This function box lets you toggle between a "Linear" and a "Step" background.

- Click the BACKGROUND LINEAR function box to choose which type of background you want when the peak is fit.

VARY WD-determines whether the width of the peak will be allowed to vary during the fitting of the peak. This function box lets you toggle between a "Varying" and a "Fixed" width.

- Click the VARY WD function box to choose a varying or fixed width when the peak is fit.

FIT-initiates a fit of a marked peak or peaks to a gaussian function.

- Click the FIT function box.

This function will take 1-10 seconds to perform depending on the number of points in the fit and the number of peaks being fit. As each fit is made, the results of that fit will be scrolled in the "fit results display area," which has enough space to scroll the results from five photopeak fits. If more than five fits are made, the top line will scroll off the screen and cannot be recovered. You may make as many fits as you want, but you cannot change the region of the spectrum being viewed in MARK FIT. Change the spectrum view with the GINA selection screen.

SAVE FIT-saves the numeric results of all peaks that were fit during the last fit process.

- $\quad$ Click the SAVE FIT function box.

Even if results from previous fits are displayed in the "fit results display area," the SAVE FIT function will save only the last fit.

You must click the SAVE, FIT function box to save the results for printing.

HARD COPY-prints a plot of the fit on a laser printer.

QUIT-retums you to the GINA selection screen.

- When you have finished, click the QUIT function box. 


\subsection{Display Peak Fits (XTP)}

You may use the XTP program to display a spectrum that has been fit. XTP uses the same "peak find" and "fit" algorithms used by GNUL and displays the results of the peak fits.

XTP will access those spectral files located in the directory pointed to by the logical SLOT\$SPC. If this logical points to another node on a network, the files on that node can be accessed. To obtain hardcopy plots of the fit results in XTP, the logical XTP\$QUE must be defined and point to a laser printer queue.

- $\quad$ Enter 'XTP' and press <cr $>$ to start the XTP program.

The XTP will display the XTP menu, which consists of three fields-SPECTRUM SLOT, ISOTOPE LIBRARY NAME, and REQUIRED ENERGY LIBRARY. If you want to change any of the default values:

- Press $<c r>$ to move the cursor to the value you want to change.

- Enter the new value, which will appear to the right of the current value. For each menu item, only a few values are valid:

- $\quad$ SPECTRUM SLOT - the name of the spectrum file (e.g., A1).

- ISOTOPE LIBRARY - the name of the isotope library to use in the analysis.

The directory pointed to by the logical GE\$ISOTOPE\$LIB contains the isotope libraries.

- REQUIRED ENERGY LIBRARY - the name of the required energy library to use in the analysis.

The required energy library contains a list of radionuclide line energies for which the analysis code will be required to determine values for. The directory pointed to by the logical GE\$LIMIT \$LIB contains the required energy libraries.

- Press <cr $>$ to replace the old value with the new one.

- When you have finished, press $\langle\mathrm{Ctrl} \mathrm{Z}\rangle$ to save the values.

The XTP program will display the spectrum plot with the fit peaks and their corresponding energies (see Figure 5-7). Also, you will see the decade control window with a red line that shows the current decade values.

\subsubsection{Display and Print Peak Flts.}

- Click on the region of the spectrum you want to zoom in on.

- XTP will display 256 channels, centered at the location you clicked. XTP will display a fit only if all values of the fit can be displayed on the screen.

- $\quad$ Click the PRINT function box to print that spectrum region and the fit functions.

- $\quad$ Click the DISPLAY ALL function box to display the complete spectrum (see Figure 5-8). 


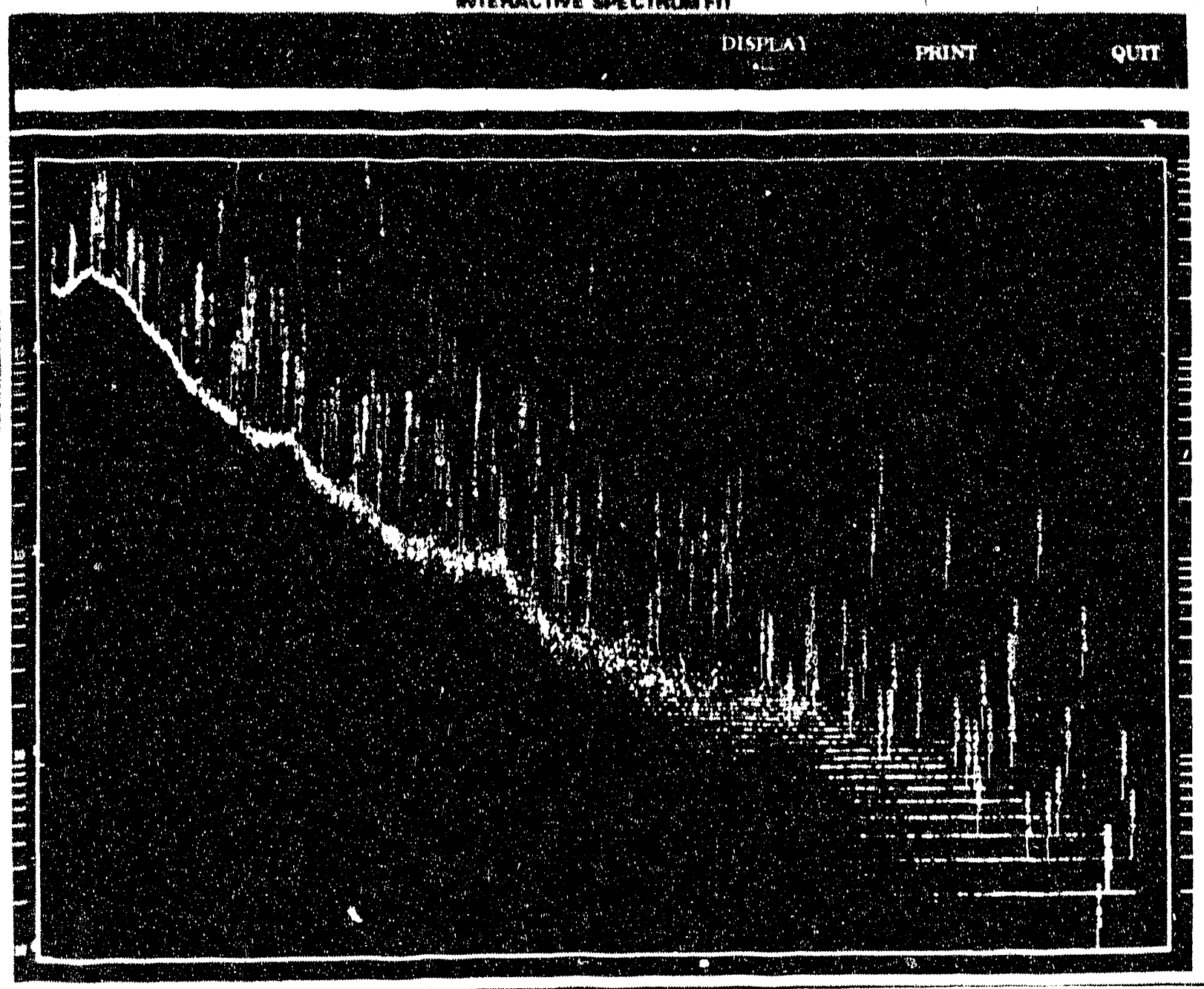

FIgure 5-7. XTP display screen, showing energy values for all possible peaks.

5.4.2 Control Decades. In the decade control window, the red bar shows how many decades are on the $\mathrm{Y}$-axis of the spectrum. You may change the value of the base decade by adding decades to and removing decades from the bottom of the red bar. Also, you may add decades to and remove decades from the top of the red bar.

To remove a decade:

- $\quad$ Click on the portion of the red bar you want to remove.

To add a decade:

- Place the cursor beyond the end of the red bar.

- Click to extend the number of decades out to the position of the cursor. 
WTE HACTNE SPE CTRUM FI
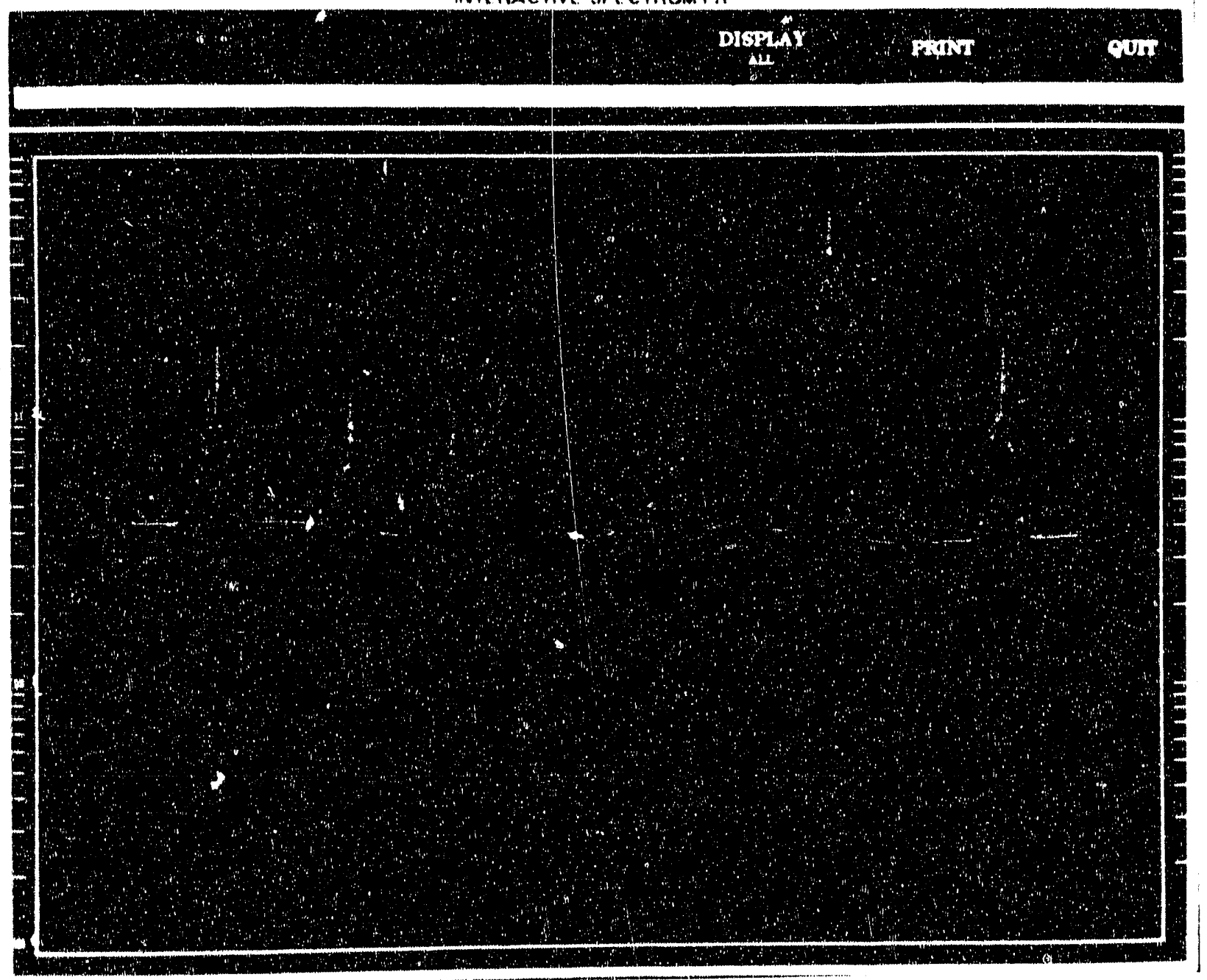

Figure 5-8. XTP spectrum screen, showing the complete spectrum. 


\section{Appendix A \\ Command Summary for VAXGAP}




$$
\text { A-2 }
$$




\section{Appendix A}

\section{Command Summary for VAXGAP}

The following is a list of the commands you may use within the VAXGAP environment.

Command Description

ADCS*TATUS Print the status of all ADCs connected to the VAX through a Canberra MCA

BACK Analyze an ambient room background sample.

CBAG Perform general spectrum analysis using concurrent background.

CBAT Perform a concurrent background analysis.

DAS Display spectrum and detector system status on a windowed terminal.

DIRHEAD Get directory listing with header information.

ECA Analyze an efficiency curve reference standard calibration source.

ECB Edit a calibration block.

EFF Create, edit, and print efficiency tables.

EPB Edit a parameter block.

GAP Perform general Ge spectrum analysis with upper limits.

GINA Perform interactive peak fitting and display on a windowed terminal.

GNUL Perform general Ge spectrum analysis with no upper limits.

$\mathrm{HEAD} \quad$ Enter spectrum header parameters and copy spectrum from disk slot to disk save area.

HP Perform unquantified spectrum analysis using GNUL fit algorithms.

MAKE_LIB Construct an isotope library from the referenced data base.

NIL Create an isotope library from text file.

PEQ Determine the pulser energy equivalents for pulsers.

PLOT Plot a spectrum on a laser printer or VT330 CRT.

PRBACK Print the ambient background for a particular spectrum slot. 
Command Description

PRDATA

Print channel contents of a spectrum on either the line printer or the CRT.

PRH*EAD

Print the spectrum header information.

PRLIB

Print the contents of an isotope library.

$\$ 90$

Transfer spectrum from Canberra MCA to a spectrum slot.

SCREEN

Spectrum analysis of samples for Department of Transportation shipping requirements.

SPECTRA

Move spectra to and from disk and magnetic tape.

STA

Start spectrum data acquisition.

STO

Stop spectrum data acquisition.

THO*R

Energy calibrate spectrum slot from a Th-228 source.

UCLB

Calibrate spectrum slot using operator input.

XTP

Display spectrum analysis and fit results for windowed terminals. 


\section{Appendix B \\ Logical Assignments in VAXGAP}

B-1 
B-2

王 


\section{Appendix B \\ Logical Assignments in VAXGAP}

The following list describes the logical assignments made for the VAXGAP system.

Directory or

Terminal Line

Description

BATCH\$RESULTS

BATCH\$RESULTS\$DIR

EFFCRV\$RESULTS

EFFICIENCY\$TABLES

GAMMA\$STD

GAP\$DISK

GAP\$EXE

GAP\$LIB

GAP\$SRC

GE\$INTERF\$LIB

GE\$ISOTOPE\$LIB

GE\$LIMIT\$LIB

GE\$PD\$LIB

GE\$SAVE, . .

LIB \$SRC

LN03

SLOT\$BKG

SLOT\$SPC
Batch analysis results files

The name of the directory file for batch analysis results

Efficiency curve analysis results files

All efficiency tables

Efficiency curve standards files

Name of the disk containing VAXGAP directories

VAXGAP executable files

VAXGAP subroutine library

VAXGAP source and object files

Interference decontamination files

Isotope library files

Limit library files

Parent-daughter correction files

Spectrum files

VAXGAP subroutine library source and object files

Terminal line to which the laser printer is connected

Background results files for all detector slots in the system

Detector slot files 


\section{Appendix C \\ Spectrum Parameter Block Contents}

C-1 
C-2 


\section{Appendix C}

\section{Spectrum Parameter Block Contents}

The following is a list of the contents of the parameter block of a Ge spectrum.

Variable

LTIME

RTIME

START_.TIME

START_DATE

S_NUM

COLLECT_TIME

COLLECT_DATE

COLLECT_HOUR

SMPL_FRACTION

EFF_MULT

EFF_TABLE

DISTANCE

VOLUME

VOLUME_UNIT'S

NO_CHANNELS

TOTAL

SYS_FLOW

SYS_VOLUME

SYS_VOLUME_UNITS

POWER
Contents

LIVE TIME IN CENTI_SECONDS

REAL TIME IN CENTI-SECONDS

TIME OF START OF COUNT

DATE OF START OF COUNT

SAMPLE NUMBER

SAMPLE COLLECTION TIME

SAMPLE COLLECTION DATE

COLLECTION DURATION (HOURS)

SAMPLE FRACTION

EFFICIENCY CORRECTION FACTOR

EFFICIENCY TABLE NUMBER

SOURCE TO DETECTOR DISTANCE

VOLUME/WEIGHT OF SAMPLE

UNITS OF VOLUME/WEIGHT

NUMBER OF CHANNELS IS SPECTRUM

TOTAL NUMBER OF COUNTS IN SPEC.

SYSTEM FLOW RATE (VOLUME/MIN)

SYSTEM VO.'JUME

UNITS FOR SYS_FLOW/SYS_VOLUME

REACTOR POWER MW 


\begin{tabular}{|c|c|}
\hline Variable & Contents \\
\hline COLL_POS & COLLIMATOR POSITION \\
\hline ERR_EFF & ERROR IN EFFICIENCY \\
\hline ERR_GEO & ERROR IN GEOMETRY \\
\hline ERR_OTH & ERROR IN OTHER \\
\hline MCA_SYSTEM & MCA SYSTEM $(\mathrm{A} 1, \mathrm{~A} 2, \ldots \mathrm{B} 1, \ldots)$ \\
\hline ADC_NUM & ADC NUMBER \\
\hline ZERO_OFFSET & ZERO ENERGY CROSS OVER \\
\hline EA_CO & A COEFFICIENT OF ENERGY EQU. \\
\hline EB_CO & B COEFFICIENT OF ENERGY EQU. \\
\hline EC_CO & C COEFFICIENT OF ENERGY EQU. \\
\hline WA_CO & A COEFFICIENT OF WIDTH EQU. \\
\hline WB_CO & B COEFFICIENT OF WIDTH EQU. \\
\hline ERR_MX(6) & ERROR MATRIX TERMS \\
\hline SA_SUM & A COEFF. RANDOM SUM EQUATION \\
\hline SB_SUM & B COEFF. RANDOM SUM EQUATION \\
\hline SC_SUM & C COEFF. RANDOM SUM EQUATION \\
\hline NO_PULSER & NUMBER OF PULSERS USED \\
\hline PULSER_RATE & PULSE RATE OF PULSER \\
\hline PLS_ONE_KEV & ENERGY OF PULSER \#1 \\
\hline PLS_TWO_KEV & ENERGY OF PULSER \#2 \\
\hline PLS_CHANNELS & NO. OF CHANNELS IN PULSER BUFF. \\
\hline PLS_ONE_START & START CHANNEL OF FIRST PULSER \\
\hline PLS_TWO_START & START CHANNEL OF SECOND PULSER \\
\hline PLS_ONE_PS & START CHANNEL OF PULSER \#1 IN PULSER SPECTRUM \\
\hline PLS_TWO_PS & START CHANNEL OF PULSER \#2 IN PULSER SPECTRUM \\
\hline
\end{tabular}


Variable

CAL_PULSER_WD

LOW_PULSER_WD

EFF_FILE
Contents

WID'TH OF PULSER AT CALIBRATION

WIDTH OF CURRENT LOW PULSER

EFFICIENCY TABLE FILE NAME 
Appendix D

File Structure for Ge Spectra

D-1 


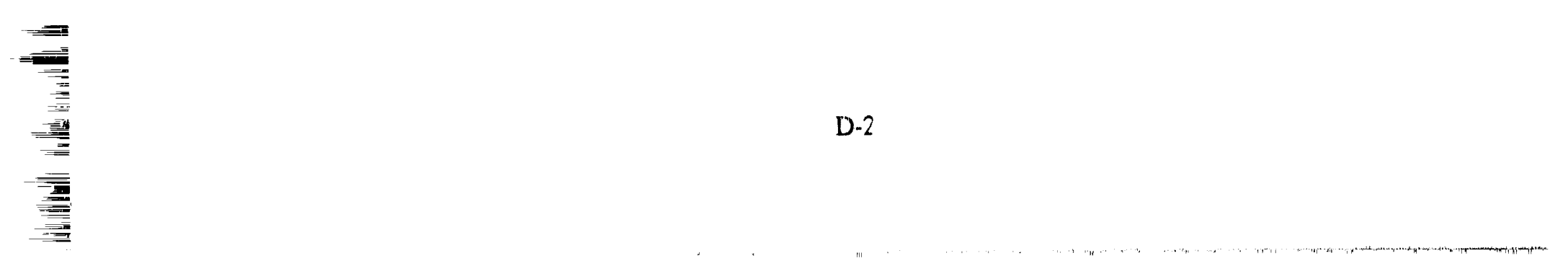




\section{Appendix D \\ File Structure for Go Spectra}

This appendix shows the file format for the storage of gamma-ray pulse height spectra on the GAP disk.

512-byte blocks:

\begin{tabular}{|c|c|c|}
\hline ID Block & - & 1 block \\
\hline Parameter Block & - & 1 block \\
\hline Pulser Spectrum & - & 4 blocks \\
\hline Gamma-ray Spectrum & - & $\begin{array}{l}64 \text { blocks for } 8 \mathrm{~K} \text { spectrum } \\
32 \text { blocks for } 4 \mathrm{~K} \text { spectrum }\end{array}$ \\
\hline
\end{tabular}

\section{ID Block:}

The information stored in the ID block is ASCII character data only: The first 60 bytes are reserved for spectrum ID numbers of 3 fields of 20 bytes in each field. The remaining bytes will contain spectrum header information.

Parameter Block:

The items stored in this block are as defined in the magnetic tape standard. All items will be stored in binary format (see Appendix A).

Pulser Spectrum:

The pulser spectrum will be stored here. Enough room exists for 512 channels of pulse height data. The channel contents will be in floating point format.

Garnma-ray Spectrum:

The Gamma-ray spectrum block will contain the pulse height spectrum in blocks of 128 channels. The channel contents will be in floating point format. 


\section{Appendix E \\ Description of "Flag" Values- \\ Printed as Part of Isotope Summary}


$=$ 焉

$=$

E-2 


\section{Aippendix E \\ Description of "Flag" Values- \\ Printed as Part of Isotope Summary}

This appendix describes the flag values that are printed with each line during the isotope summary printout obtained from an analysis of a Ge spectrum. The net value printed could be a combination of values given below:

Flag Value

Description

\begin{aligned} & \hline 0 Peak fit by nonlinear fitting process \\ & 1 Peak fit by linear fitting process \\ & 2 Peak fit by nonlinear fitting process as part of multiplet \\ & 3 Peak fit by linear fitting process as part of multiplet \\ & 5 Peak fit by linear fitting process "upper-limit" result \\ & 7 Peak fit by linear fitting process as part of multiplet "upper-limit" result \\ & 8 Background corrected \\ & 12 Peak that may be a coincidence sum peak \\ & 16 Background corrected "upper-limit" result \\ & 32 Interference decontaminated \\ & 64 Interference decontaminated "upper-limit" result \\ & 128 Peak listed as clean in interference library \\ & 256 Line value converted to "upper-limit" in CLEANUP \\ & 512 Line is an "outlier" not used in weighted average \\ & \hline\end{aligned}

As an example of flag values being summed together: A net flag value of $554(2+8+32+512)$ indicates the following:

1. The initial peak was fit as multiplet by GAUSS.

2. The peak counts/second was corrected for a background radiation level

3. The peak counts/second was interference-decontaminated

4. The peak value was sejected as an outlier and was not used in the al weighted average. 

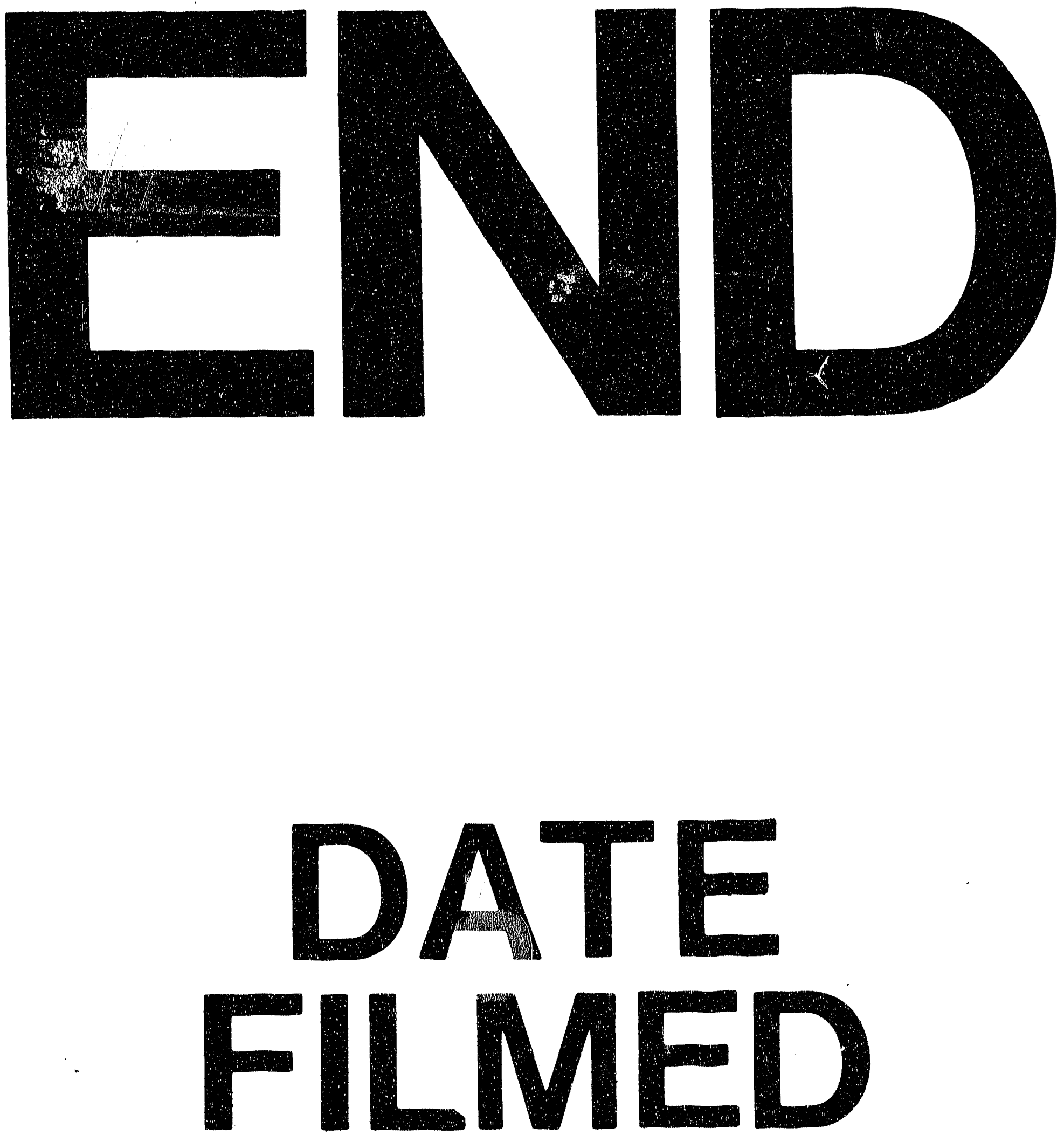

㢲

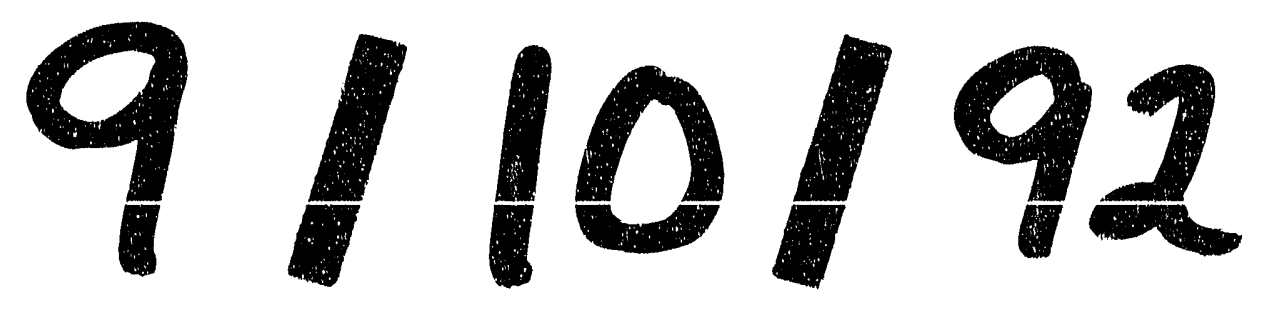




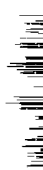

